\title{
Development
}

\section{Circuit-Specific Dendritic Development in the Piriform Cortex}

\author{
Laura Moreno-Velasquez, ${ }^{1}$ - $H u n g$ Lo, ${ }^{1,5}$ DStephen Lenzi, ${ }^{3}$ Malte Kaehne, ${ }^{2}$ Jörg Breustedt, ${ }^{1}$ \\ (D) Dietmar Schmitz, ${ }^{1,4,5}$ Sten Rüdiger, ${ }^{3}$ and Friedrich W. Johenning ${ }^{1,5}$
}

\author{
https://doi.org/10.1523/ENEURO.0083-20.2020
}

\begin{abstract}
${ }^{1}$ Neuroscience Research Center, Charité-Universitätsmedizin Berlin, Corporate member of Freie Universität Berlin, Humboldt-Universität zu Berlin, and Berlin Institute of Health, 10117 Berlin, Germany, ${ }^{2}$ Institute of Physics, Humboldt Universität Berlin, 12489 Berlin, Germany, ${ }^{3}$ Sainsbury Wellcome Centre, University College London, London W1T 4JG, UK, ${ }^{4}$ NeuroCure Cluster of Excellence, 10117 Berlin, Germany, and ${ }^{5}$ Einstein Center for Neurosciences Berlin, 10117 Berlin, Germany
\end{abstract}

\begin{abstract}
Dendritic geometry is largely determined during postnatal development and has a substantial impact on neural function. In sensory processing, postnatal development of the dendritic tree is affected by two dominant circuit motifs, ascending sensory feedforward inputs and descending and local recurrent connections. In the three-layered anterior piriform cortex (aPCx), neurons in the sublayers $2 a$ and $2 b$ display vertical segregation of these two circuit motifs. Here, we combined electrophysiology, detailed morphometry, and $\mathrm{Ca}^{2+}$ imaging in acute mouse brain slices and modeling to study circuit-specific aspects of dendritic development. We observed that determination of branching complexity, dendritic length increases, and pruning occurred in distinct developmental phases. Layer 2a and layer $2 \mathrm{~b}$ neurons displayed developmental phase-specific differences between their apical and basal dendritic trees related to differences in circuit incorporation. We further identified functional candidate mechanisms for circuit-specific differences in postnatal dendritic growth in sublayers $2 a$ and $2 b$ at the mesoscale and microscale levels. Already in the first postnatal week, functional connectivity of layer $2 a$ and layer $2 b$ neurons during early spontaneous network activity scales with differences in basal dendritic growth. During the early critical period of sensory plasticity in the piriform cortex, our data are consistent with a model that proposes a role for dendritic NMDA-spikes in selecting branches for survival during developmental pruning in apical dendrites. The different stages of the morphologic and functional developmental pattern differences between layer $2 \mathrm{a}$ and layer $2 \mathrm{~b}$ neurons demonstrate the complex interplay between dendritic development and circuit specificity.
\end{abstract}

Key words: dendritic development; NMDA-spike; piriform cortex

\section{Significance Statement}

Sensory cortices are composed of ascending sensory circuits that relay sensory information from the periphery and recurrent intracortical circuits. Dendritic trees of neurons are shaped during development and determine which circuits contribute to the neuronal input space. To date, circuit-specific aspects of dendritic development and the underlying mechanisms are poorly understood. Here, we investigate dendritic development in layer 2 of the piriform cortex, a three-layered palaeocortex that displays a clear vertical segregation of sensory and recurrent circuits. Our results suggest that dendritic development occurs in distinct developmental phases with different circuit-specific properties. We further identify candidate mechanisms for neuronal activity patterns that could determine differences in circuit-specific dendritic development.

Received March 3, 2020; accepted April 20, 2020; First published May 26, 2020.

The authors declare no competing financial interests.
Author contributions: L.M.-V., H.L., S.L., M.K., J.B., D.S., S.R., and F.W.J. designed research; L.M.-V., H.L., S.L., M.K., and F.W.J. performed research; L.M.-V., H.L., S.L., M.K., J.B., S.R., and F.W.J. analyzed data; L.M.-V., M.K., J.B., D.S., S.R., and F.W.J. wrote the paper. 


\section{Introduction}

The complex geometry of neuronal dendritic trees in relation to their function is not yet fully understood. In sensory cortices, sensory input from the periphery is distributed to cortical neurons in an ascending sensory stream of input. Recurrent connectivity between cortical neurons constitutes the local and descending stream of input, which then transforms the sensory input into cortical output (Srinivasan and Stevens, 2018; Kanari et al., 2019). Developmental growth patterns of dendritic structures are an important determinant of a neuron's function within the different circuits constituting its synaptic input space (Lanoue and Cooper, 2018). This brings up the question of how dendritic morphology develops in relation to the two different glutamatergic circuit elements in sensory information processing, ascending sensory input and recurrent connectivity.

We investigated the palaeocortical three-layered anterior piriform or primary olfactory cortex (aPCx), which shares structural and functional similarities with the reptilian dorsal cortex (Fournier et al., 2015). The aPCx is the largest cortical region receiving olfactory sensory inputs. Peripheral odor information from nasal olfactory sensory neurons converges onto the aPCx via the olfactory bulb. Functionally, the aPCx synthesizes the segregated peripheral input into odor objects and identifies them (Wilson and Sullivan, 2011). Unlike topographically organized neocortical sensory systems, afferent sensory and recurrent input streams to the aPCx lack any apparent spatial structure and are therefore non-topographical (Srinivasan and Stevens, 2018). Layer 2 is the main cellular layer of the olfactory cortex (Bekkers and Suzuki, 2013). Based on the distribution of genetic markers and somatic morphology, layer 2 can be divided into layer $2 a$ (superficial third) and layer 2b (deeper two-thirds; Diodato et al., 2016; Choy et al., 2017; Bolding et al., 2019; MartinLopez et al., 2019). Layer $2 a$ predominantly contains superficial so-called semilunar cells (layer $2 a$ neurons). Layer $2 \mathrm{~b}$ harbors pyramidal cells and semilunar-pyramidal transition cells (layer 2b neurons; Suzuki and Bekkers, 2011; Choy et al., 2017). Neurons in the two sublayers display differences in functional circuit incorporation. Layer 2a neurons predominantly sample converging sensory input and distribute it unidirectionally to the layer $2 \mathrm{~b}$ and 3 neurons. Layer $2 b$ neurons receive sensory input

This work was supported by the Deutsche Forschungsgemeinschaft (German Research Foundation) Grants SFB 1315 Project-ID 327654276, Exc 2049, and SPP 1665 (to D.S.); JO 1079/3-1 (to F.W.J.); and RU1660/5-1 (to S.R.); the Bundesministerium für Bildung und Forschung (Federal Ministry of Education and Research) Grant 01GQ1420B (to D.S.); and the ERC BrainPlaySynergy Grant (D.S.).

Acknowledgements: We thank Robert Sachdev and Matthew Larkum for the Ai95 mouse line. We also thank Anke Schönherr and Susanne Rieckmann for excellent technical assistance.

Correspondence should be addressed to Friedrich W. Johenning at friedrich.johenning@charite.de.

https://doi.org/10.1523/ENEURO.0083-20.2020

Copyright (C) 2020 Moreno-Velasquez et al.

This is an open-access article distributed under the terms of the Creative Commons Attribution 4.0 International license, which permits unrestricted use, distribution and reproduction in any medium provided that the original work is properly attributed. and, in addition, are incorporated in a rich recurrent network (Suzuki and Bekkers, 2011; Wiegand et al., 2011; Hagiwara et al., 2012; Choy et al., 2017). Recently, it has been demonstrated in vivo that these two neuron types play different roles in reading out converging sensory input (layer 2 a neurons) and performing pattern storage and completion via recurrent circuits (layer $2 \mathrm{~b}$ neurons; Bolding et al., 2019).

This vertical organization of input space of layer 2 neurons extends to the dendritic tree, where sensory and recurrent functional domains are spatially segregated. In the apical dendrites of all neurons in layer 2, the majority of sensory input projects to the superficial layer 1a. Layer 1a can be clearly distinguished from layer $1 \mathrm{~b}$, which, together with inputs in layers 2 and 3, samples recurrent inputs. Basal dendrites exclusively sample recurrent inputs (Franks Kevin and Isaacson, 2005; Johenning et al., 2009). In aPCx, we therefore observe a clear vertical segregation of functionally distinct cell types and of different functional dendritic domains. This feature of aPCx makes layer 2 of the aPCx an ideal model for the differential analysis of dendritic growth patterns related to sensory input and recurrent connectivity.

Here, we studied developmental dendritic growth in layer $2 \mathrm{a}$ and layer $2 \mathrm{~b}$ neurons in acute brain slices of the aPCx. We applied electrophysiology, detailed morphometry of 3D-reconstructed neurons, $\mathrm{Ca}^{2+}$ imaging and computational modeling. We identified distinct phases of dendritic development with cell-type-specific differences of dendritic growth and pruning patterns. We related the different developmental patterns described here at the morphologic level to physiological differences at the microscale and mesoscale levels. This enabled us to identify candidate mechanisms that may drive circuit-specific dendritic development in a non-topographic sensory system.

\section{Materials and Methods}

\section{Slice preparation}

Acute brain slices were prepared from C57BI6N mice of either sex except for population $\mathrm{Ca}^{2+}$ imaging experiments with GCaMP, where Ai95-NexCre mice were used. In experiments for Figures 1-5, the horizontal slicing orientation was chosen to preserve rostrocaudal association fibers (Demir et al., 2001). For layer 1a dendritic spike measurements in Figure 6, we used coronal slices. All animal procedures were performed in accordance with the national and institutional guidelines of the Charité-Universitätsmedizin Berlin. All procedures were approved by the local health authority and the local ethics committee (Landesamt für Gesundheit und Soziales, Berlin). For morphologic reconstruction, acute brain slices were prepared at four age intervals expressed in postnatal days (p): p1-p2, p6-p8, p12-p14, and p30-p40 (>p30). Electrophysiological Characterization was limited to the two age intervals p12p14 and p30-p40. For measurements of NMDA-spikes, coronal slices were prepared at p14-p21. Brains from p30p40 mice and from mice used for dendritic spike measurements were prepared in ice-cold artificial CSF (ACSF; pH 
$\mathrm{A}_{1}$

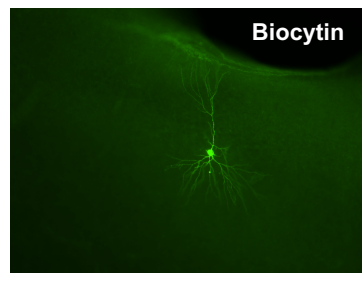

$\mathbf{B}_{1}$ Layer 2A neurons

$1-2 \mathrm{pd}$

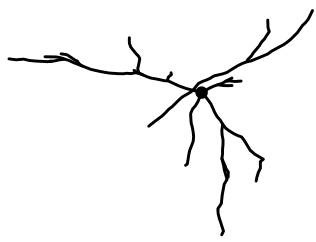

$\mathbf{B}_{2}$ Layer 2B neurons

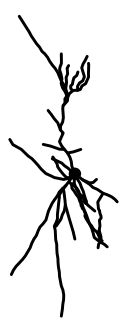

$A_{2}$

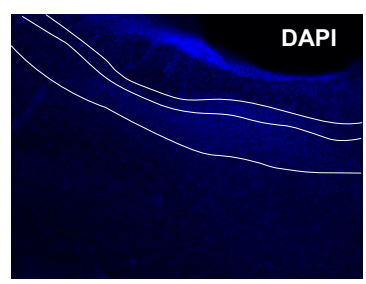

$6-8 \mathrm{pd}$
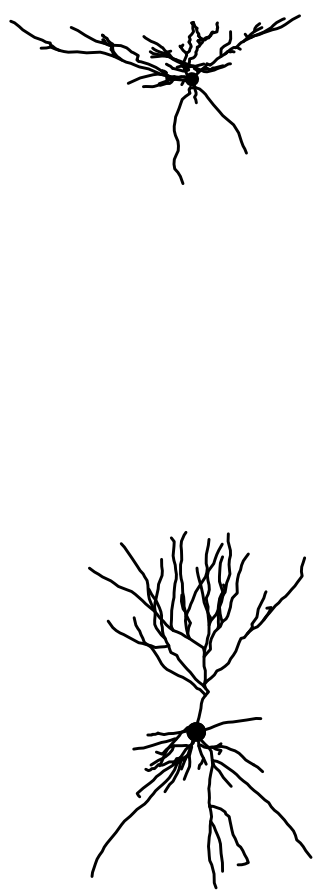

$\mathbf{C}_{2}$

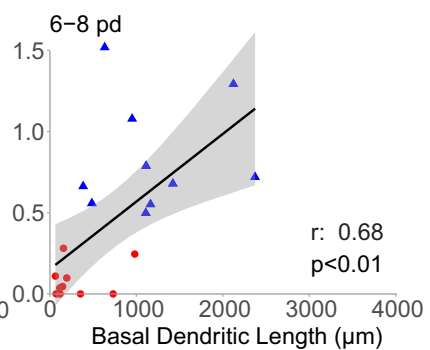

Basal Dendritic Length $(\mu \mathrm{m})$
$A_{3}$

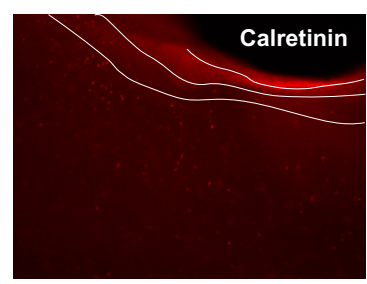

$12-14 \mathrm{pd}$

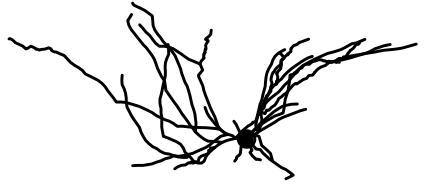

$\mathbf{A}_{4}$

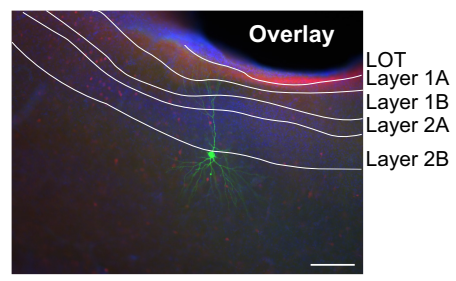

$>30 \mathrm{pd}$

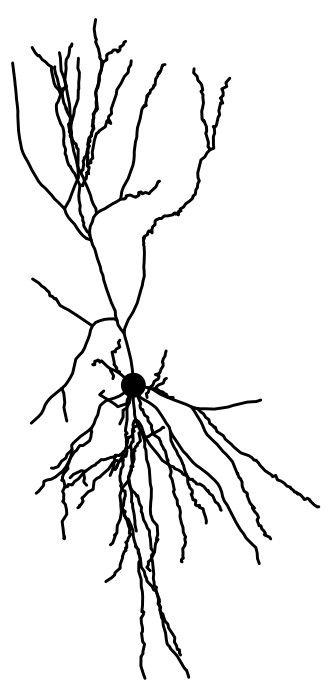

$\mathrm{C}_{3}$

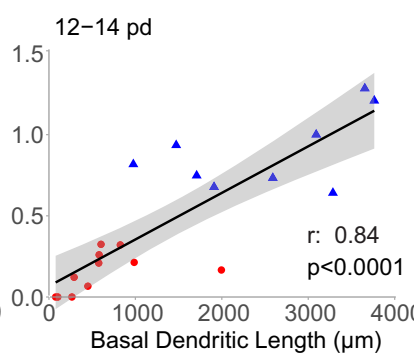

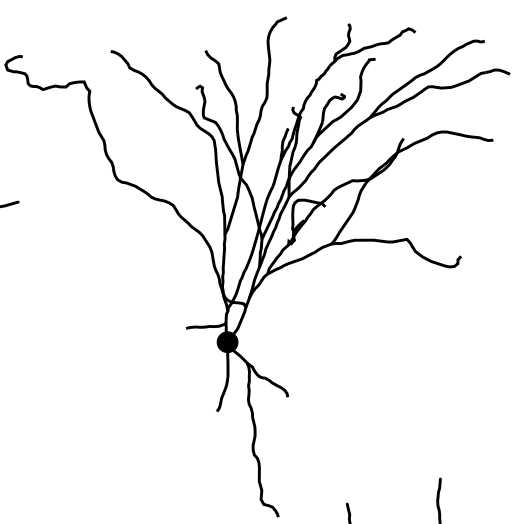

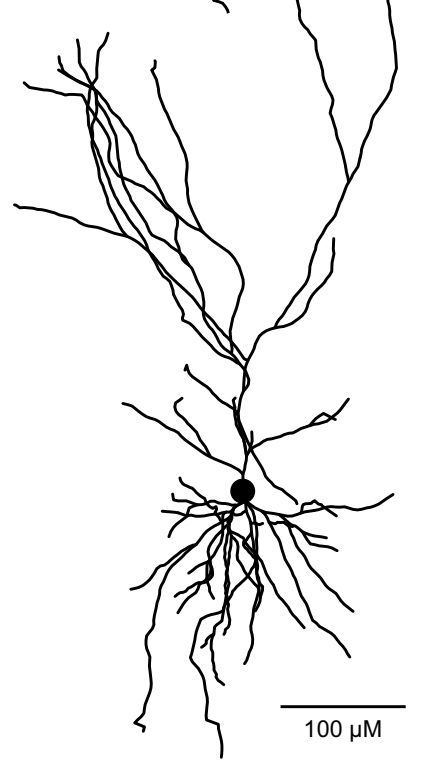

$\mathbf{C}_{1}$

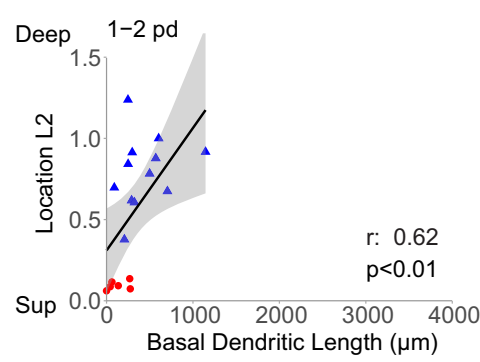

$\mathbf{C}_{4}$

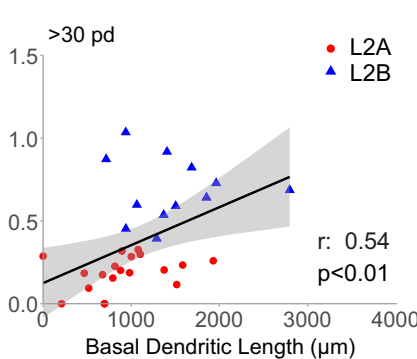

Figure 1. Localization and differentiation of the two principal neuron types in layer 2 of the aPCx. $\boldsymbol{A}$, Slice containing one recorded neuron filled with biocytin (A1) and, additionally, post hoc labeled with DAPI (A2) and calretinin (A3). The overlay (A4) shows the recorded neuron located in the layer $2 \mathrm{~b} /$ layer 3 transition zone of the aPCx. LOT stands for lateral olfactory tract. $\boldsymbol{B}, 3 \mathrm{D}$ morphologic reconstructions of different layer $2 a(B 1)$ and layer $2 b($ B2) neurons at four time windows (expressed as postnatal days, pd): right 
continued

after birth (p1-p2), at the end of the first postnatal week (p6-p8), at the end of the second postnatal week (p12-p14), and after the fifth postnatal week $(>\mathrm{p} 30)$. Scale bar: $100 \mu \mathrm{m}$. C Correlation between the total basal dendritic length and the vertical position of the cells in layer 2 (layer 2a neurons (L2A) in red, layer 2b neurons (L2B) in blue) is shown at the same four time windows (C1: p1-2, C2: p6-8, C3: p12-14, C4: >p30). Spearman $r$ and $p$ values are shown for each time group. See Extended Data Figure 1-1 for further details.

Figure Contributions: Laura Moreno-Velasquez performed the experiments and analyzed the data.

7.4) containing the following: $87 \mathrm{~mm} \mathrm{NaCl}, 26 \mathrm{~mm} \mathrm{NaHCO}_{3}$, $10 \mathrm{~mm}$ glucose, $2.5 \mathrm{~mm} \mathrm{KCl}, 3 \mathrm{~mm} \mathrm{MgCl}, 1.25 \mathrm{~mm}$ $\mathrm{NaH}_{2} \mathrm{PO}_{4}, 0.5 \mathrm{~mm} \mathrm{CaCl}$, and $50 \mathrm{~mm}$ sucrose. Slices were cut at $400-\mu \mathrm{m}$ thickness and incubated at $35^{\circ} \mathrm{C}$ for $30 \mathrm{~min}$. The slices were then transferred to standard ACSF containing the following: $119 \mathrm{~mm} \mathrm{NaCl}, 26 \mathrm{~mm} \mathrm{NaHCO}, 10 \mathrm{~mm}$ glucose, $2.5 \mathrm{~mm} \mathrm{KCl}, 2.5 \mathrm{mM} \mathrm{CaCl}_{2}, 1.3 \mathrm{mM} \mathrm{MgCl}_{2}$, and 1 $\mathrm{mm} \mathrm{NaH} \mathrm{PO}_{4}$. Slices from other age groups were cut in ice-cold standard ACSF and incubated for $30 \mathrm{~min}$ in standard ACSF at $35^{\circ} \mathrm{C}$. The slices were then stored in standard ACSF at room temperature in a submerged chamber for $0.5-6 \mathrm{~h}$ before being transferred to the recording chamber. For dendritic spike measurements in Figure 6, $1 \mu \mathrm{m}$ gabazine was added to the recording solution. Experiments requiring spontaneous network activity were prepared in icecold ACSF containing the following: $125 \mathrm{~mm} \mathrm{NaCL}, 25 \mathrm{~mm}$ $\mathrm{NaCHCO}_{3}, 10 \mathrm{~mm}$ glucose, $4 \mathrm{~mm} \mathrm{KCL}, 1.25 \mathrm{~mm} \mathrm{NaH}_{2} \mathrm{PO}_{4}$, $2 \mathrm{mM} \mathrm{CaCl}_{2}$, and $1 \mathrm{mM} \mathrm{MgCl}_{2}$. Slices were incubated at $35^{\circ}$ $\mathrm{C}$ for $30 \mathrm{~min}$ and stored at room temperature in a submerged chamber for 0.5-7 $\mathrm{h}$. All recordings were performed at near-physiological temperature $\left(32-34^{\circ} \mathrm{C}\right)$.

\section{Electrophysiology}

Whole-cell current clamp experiments were performed at near physiological temperature $\left(32-34^{\circ} \mathrm{C}\right)$ using an Axon Multiclamp 700B amplifier (Molecular Devices). For morphologic reconstruction and characterization, signals were low pass filtered at $2 \mathrm{kHz}$ and digitized at a sampling rate of $20 \mathrm{kHz}$ (BNC-2090, National Instruments Corporation). Pipettes (3-6 M $\Omega$ ) were filled with an intracellular solution containing the following: $135 \mathrm{~mm} \mathrm{~K}$-gluconate, $6 \mathrm{~mm} \mathrm{KCl}$, 10 mм HEPES, 0.2 mм EGTA, 2 mм $\mathrm{MgCl}_{2}$, 2 mм Na-ATP, $0.5 \mathrm{~mm} \mathrm{Na}-\mathrm{GTP}$, and $5 \mathrm{~mm}$ phosphocreatine $\mathrm{Na}(\mathrm{pH} 7.3)$ and biocytine $(0.20 \%)$. Liquid junction potential (LJP) was not corrected. Bridge balance compensation was applied in current clamp. Cells were discarded if the resting membrane potential was above $-60 \mathrm{mV}$ or the series resistance exceeded $30 \mathrm{M} \Omega$. For dendritic spike recordings, signals were low pass filtered at $8 \mathrm{kHz}$ and digitized at a sampling rate of $20 \mathrm{kHz}$. Pipettes (3-6 M 2 ) were filled with an intracellular solution containing the following: $130 \mathrm{~mm}$ K-gluconate, $20 \mathrm{~mm} \mathrm{KCl}, 10 \mathrm{~mm}$ HEPES, $4 \mathrm{~mm}$ MgATP, $0.3 \mathrm{~mm}$ NaGTP, $10 \mathrm{~mm}$ phosphocreatine $(\mathrm{pH} 7.3$, adjusted with $\mathrm{KOH}$ ), $30 \mu \mathrm{m}$ Alexa Fluor (AF) 594, and $500 \mu \mathrm{m}$ fluo-5F. Experiments were conducted without exceeding $-200 \mathrm{pA}$ at resting membrane potential (layer 2a neurons were held at $-60 \mathrm{mV}$, and layer $2 \mathrm{~b}$ neurons were held at $-70 \mathrm{mV}$ ). Series resistance was below $30 \mathrm{M} \Omega$. After dye-filling the patched neuron for $10 \mathrm{~min}$, we placed the $\theta$-glass stimulation electrode close to the distal dendrite in layer 1a of the piriform cortex. Stimulation protocol was set to three pulses at $50 \mathrm{~Hz}$ with $10-\mu \mathrm{A}$ steps (with one exception in layer $2 \mathrm{~b}$ neuron, which was with $20-\mu \mathrm{A}$ steps).

\section{Electrophysiological analysis}

Analysis was performed using custom-written routines in Python. Resting membrane potential $(\mathrm{Vm})$ was taken as the mean value of the baseline before current injections were performed. For characterization, neurons were held at $-60 \mathrm{mV}$. Input resistance (IR), membrane time constant (Tau), and membrane capacitance $(\mathrm{Cm})$ were calculated from the voltage response to an 80-pA hyperpolarizing current step. Action potential (AP) threshold was defined as the membrane potential at the point where the slope $(\mathrm{dV} / \mathrm{dt})$ reached $1 \%$ of its maximum. The fast after-hyperpolarization (fAHP) was defined as the difference between AP threshold and the minimum voltage seen immediately after the AP peak (within $5 \mathrm{~ms}$ ). Finally, the instant firing frequency was defined as the frequency between the first and second AP. For comparability, these values were extracted from the first 600-ms current injection step that elicited at least nine APs. When analyzing the integrative behavior of apical dendrites in Figure 6, we analyzed the changes in EPSP size on linear increase of stimulation intensity. To quantify EPSP size, we measured the amplitude and area under curve of a 60-ms time window following the third pulse compared with baseline $(50-\mathrm{ms}$ period before stimulus). Effects of (2R)-amino-5-phosphonovaleric acid (APV) were quantified for the largest response that did not yet evoke an AP.

\section{Immunohistochemistry}

Slices with biocytin-filled cells were stored in $4 \%$ paraformaldehyde (PFA) overnight. The following day, slices were washed three times (10 min each) in PBS and incubated in a blocking solution composed of $5 \%$ normal goat serum (NGS; Biozol), 1\% Triton X-100 (Sigma), and PBS, for $3 \mathrm{~h}$ at room temperature with gentle agitation. Primary antibodies were diluted in blocking solution (2.5\% NGS, $1 \%$ Triton X-100, PBS), and slices were incubated for $72 \mathrm{~h}$ at $4^{\circ} \mathrm{C}$. Biocytin-filled neurons were labeled with a streptavidin marker conjugated to AF 488 (Invitrogen, S-32354; 1:500 dilution). Additionally, the lateral olfactory tract (LOT) and mitral cell axons in layer 1a were labeled with calretinin (anti-mouse; Millipore, MAB1568; 1:1000 dilution or anti-rabbit; SWANT, 7697; 1:4000 dilution) and interneurons with GAD 67 (anti-mouse; Millipore, MAB 5406; 1:500 dilution), GAD 65/67 (anti-rabbit; Chemicon, AB 11070; 1:500 dilution), or gephyrin (anti-mouse; SYSY $147111 ; 1: 500$ dilution).

Following this, slices were washed two times (10 min each) with PBS and secondary antibodies (goat anti-rabbit AF 555, goat anti-rabbit AF 647, goat anti-mouse AF 
555, goat anti-mouse AF 647; Invitrogen; 1:500 dilution in $0.5 \%$ Triton $\mathrm{X}-100$, PBS) were applied for $3 \mathrm{~h}$ at room temperature. Finally, slices were washed three times (10 min each) in PBS and mounted on glass slides in mounting medium Fluoroshield with 4',6-diamidino-2-phenylindole (DAPI; Sigma).

\section{Reconstructions and morphologic analysis}

Mounted slices were visualized on a fluorescent microscope $(10 \times$ objective, 0.3 N.A.; Leica) to identify and select the biocytin-filled neurons located in the aPCx for further reconstruction. Only neurons that displayed homogenous filling with biocytin and lacked obvious amputation of the dendritic tree by slicing were analyzed. Therefore, not all neurons chosen for electrophysiology were also chosen for morphologic reconstruction and vice versa. Selected slices were then imaged on an upright Leica TCS SP5 confocal microscope (Leica Microsystems) through a $20 \times$ immersion objective $(0.7$ N.A.; Leica) with 405-nm (diode), 488-nm (argon), 568-nm (solid state), and 633-nm (helium-neon) laser lines. For biocytin-filled neurons, the perisomatic field of view was further imaged through a $63 \times$ immersion objective $(1.4 \mathrm{~N}$. A.; Leica) to validate the spine density. Cells were selected and classified according to their position in layer 2 of the aPCx using FIJI (https://imagej.nih.gov/ij/). The position in layer 2 was defined as the smallest distance from the soma to the border between layer $1 \mathrm{~b}$ and layer $2 \mathrm{a}$, normalized to the total width of layer 2 for each neuron. The border between layers $1 \mathrm{a}$ and $1 \mathrm{~b}$ was traced to later classify the apical dendrites according to their synaptic inputs. Neuronal morphologies were then reconstructed with neuTube software (Feng et al., 2015) and exported as SWC files. Morphometric parameters were extracted with L-measure software (Scorcioni et al., 2008) and analyzed with $\mathrm{R}$ studio and Python using btmorph v2 (TorbenNielsen, 2014) and SciPy packages.

\section{$\mathrm{Ca}^{2+}$ imaging}

For population $\mathrm{Ca}^{2+}$ imaging of neonatal spontaneous synchronous network events (Fig. 4), we used the genetically encoded $\mathrm{Ca}^{2+}$ indicator (GECl) GCaMP6F. NEX-Cre mice (Goebbels et al., 2006) were crossed with Ai95 animals (https://www.jax.org/strain/024105; Madisen et al., 2015) for constitutive GCaMP6F expression in excitatory cells only.

For experiments involving spontaneous network activity, $\mathrm{Ca}^{2+}$ imaging was performed using a Yokogawa CSU-22 spinning disk microscope at $5000 \mathrm{rpm}$. The spinning disk confocal permitted the generation of a large field of view time series at a high acquisition rate. A 488-nm laser was focused onto the field of view using a $40 \times$ objective. Emission light was filtered using a $515 \pm 15-\mathrm{nm}$ bandpass filter. Fluorescence was detected using an Andor Ixon DU-897D back-illuminated CCD, with a pixel size of $16 \mu \mathrm{m}$. Andor iQ software was used for data acquisition. Population $\mathrm{Ca}^{2+}$ imaging was performed at $10 \mathrm{~Hz}$ when single cells were measured. For measuring of larger regions of interest (ROIs) incorporating layer $2,10-\mathrm{Hz}$ data were pooled with a dataset acquired at $40 \mathrm{~Hz}$.

For analyzing dendritic spikes using 2P-imaging, $30 \mu \mathrm{M}$ AF 594 and $500 \mu \mathrm{m}$ Fluo-5F were added to the intracellular solution. A Femto 2D two-photon laser scanning system (Femtonics Ltd.) equipped with a femtosecond pulsed Ti: Sapphire laser tuned to $\lambda=805 \mathrm{~nm}$ (Cameleon, Coherent) controlled by the MATLAB-based MES software package (Femtonics Ltd.) was used. Fluorescence was detected in epifluorescence mode with a water immersion objective (LUMPLFL $60 \times / 1.0$ N.A., Olympus). Transfluorescence and transmitted infra-red light were detected using an oil immersion condenser (Olympus). The average scanning speed was $300 \mathrm{~Hz}$, and the intermediate sections were jumped over within $60 \mu$ s using a spline interpolated path. Dendritic $\mathrm{Ca}^{2+}$ transients were measured every $30 \mathrm{~s}$.

\section{Imaging analysis}

For population $\mathrm{Ca}^{2+}$ imaging (Fig. 4), fields of view (FOVs) with at least $5 \mathrm{~min}$ of recordings were included in the analysis. Videos were motion corrected using Suite2 $p$ (Pachitariu et al., 2017) and analyzed using custom Python code and SamuROI (Rueckl et al., 2017). If motion artefacts were too great to be corrected, recordings were not included in the subsequent analysis. Each pixel of the raw data was normalized using the six-sample window with the lowest SD. Traces were extracted from each ROI, and event detection was conducted. Events were detected as increases in $\Delta F / F>2.5$ SDs from baseline with a peak width of at least two consecutive samples. The results were manually curated with the user free to exclude events based on the interevent interval, amplitude, signalto-noise ratio, and peak width. Incomplete events at the start or end of each recording were excluded from analysis. The rates of false positives and negatives were $4.5 \%$ and $5.1 \%$, respectively, calculated from a random subset of the data (100 cells, three mice).

The upper and lower boundaries of layer 2 were manually defined based on cell density. For detection of global events, we measured the average change in fluorescence for all pixels of layer 2 piriform cortex, including the neuropil, using a rectangular $\mathrm{ROI}$ defined by the upper and lower boundaries of layer 2.

For single-cell analysis, we used a semi-automated method based on image segmentation with llastik (Sommer et al., 2011). This was required because a large number of cells were inactive, or closely packed and/or synchronous in their activity. Ilastik was trained to segment z-projection sum images of a subset of FOVs to produce a five-label image (nuclei, somata, bright debris, dark debris, and background). Cells were detected using the nuclei label with false positives manually rejected. Using these cell locations, the somata image was divided into territories using watershed segmentation, and only the nearest pixels to each nucleus were included. ROls with fewer than 70 pixels were rejected. We calculated the $\Delta F / F$ for each $R O I$ and subtracted an estimate of the local neuropil contribution using an equal number of randomly selected non-cell pixels within a fixed radius of 70 pixels. 


\section{Modeling}

For the model in Figure 7, we assumed that the density of synapses on the branches of a dendrite is constant, i.e., depends linearly on the length of a branch. We assumed that on odor exposure a maximum of 70 active synapses can arrive at a single neuronal dendrite. Following recent experimental measurements (Srinivasan and Stevens, 2018), we considered 3700 glomeruli, $9.7 \times 107$ synapses between all glomeruli and all layer 2 neurons, and a total of 41,000 layer 2 neurons. Hence, we dealt with an average $9.7 \times 107 /$ $41,000=2366$ synapses between all glomeruli and one neuron. Thus, for 70 synapses to be activated on odor exposure, we assumed that 109 glomeruli are activated per odor $(=3700 \times 70 / 2366)$.

We characterized the morphology of a neuron through its mean branch length (BL), which we found to range from 40 to $110 \mu \mathrm{m}$ (mean is $72 \mu \mathrm{m}$; see Results). The total dendritic $B L$ (TDBL) on average is $\sim 1800 \mu \mathrm{m}$.

For simplicity, we approximated the length of any branch by the mean values of the respective neurons. Accordingly, dendrites could have a maximum of 1800/ $40=45$ (short-branched neuron) and a minimum of 1800/ $110=16$ branches (long-branched neuron).

We distinguished the two cases of clustered $\left(n_{\text {clus }}\right)$ and distributed $\left(n_{\text {dist }}\right)$ stimulation. We assumed that a neuron fires if it is exposed to more than $n_{\text {dist }}=40$ active input synapses or $n_{\text {clus }}=10$ active inputs arriving on the same branch. The excitation behavior is schematically illustrated in Figure $7 A$, showing a simplified version of the nonlinear dendritic integration scheme proposed by Poirazi et al. (2003). Specifically, we considered every neuron as a two-layered network that may or may not produce a network response (express a somatic AP) to a presented stimulus set. This response is triggered in cases when the distributed input reaches a certain threshold number (reflecting a number of active input synapses), which can be understood as a linear integration scheme of the neuron. In addition, the network may produce a response to a dendritic spike. In our model, dendritic spikes led to the nonlinear integration of synaptic input, which is mimicked through the activation gates on every branch of the neuron (first layer of the network). In Figure 7A, this first layer of the network is shown as blue circles. The green circle represents the soma. The magnification insets illustrate the stimulus-response relationship of the separate branches and the soma, respectively. The model was constructed such that a somatic response is expressed if the distributed stimulation crosses the threshold value $\mathrm{n}_{\text {dist }}$ or if one of the branches expresses a dendritic spike, which relates to the number of active synapses on the branch crossing the threshold number of clustered stimulation $\mathrm{n}_{\text {clus }}$.

Using this described model, we investigated the additional response probability that is introduced through considering clustered stimulation. We supposed the number of odor-activated synapses (which we refer to as $\lambda$ ) connected to a given neuron to be random. The probability of finding a neuron that is connected to $n$ or more odor activated synapses can then be approximated as

$$
P(\lambda, n)=1-\sum_{k=1}^{n-1} \frac{\lambda^{k} e^{-\lambda}}{k !} .
$$

Equation 1 allowed us to map the response probability of a neuron to a presented stimulus. In the case of clustered stimulation, this picture is slightly different: Instead of the mean number of synapses per neuron, the mean number of synapses per branch is the relevant quantity. The mean number of branches (NB) is approximately the TDBL divided by the mean BL. We took the distribution of $B L$ into account as we reasoned that longer branches optimize the input-output relationship in case of clustered stimulation. At p12-p14, the length of the dendritic branches ranged from 40 to $110 \mu \mathrm{m}$. The response probability for clustered stimulation is modeled as following:

$$
P(\lambda, n)=1-\left(1-\sum_{k=1}^{n-1} \frac{\lambda^{k} e^{-\lambda}}{k !}\right)^{\frac{\mathrm{TDBL}}{\mathrm{BL}}}, \text { with } \lambda=S \frac{\mathrm{BL}}{\mathrm{TDBL}} .
$$

It is important to note that under such a scheme, stimulation of any dendritic branch can be sufficient to excite the neuron.

\section{Code accessibility}

The code underlying the calculations and plot in Figure 7 is available as Extended Data 1 and via GitHub (https:// github.com/mkahne/DendriticBranches). The code was executed using Python 3.7.3 on a MacBook Pro running on MacOs 10.15.4.

\section{Statistics}

Data were first tested for normality. Statistical tests were performed as indicated using GraphPad Prism, the SciPy library, and the DABEST package in Python and R (Ho et al., 2019). We used the paired (normally distributed direct comparisons) and unpaired $t$ test (normally distributed single comparisons), Wilcoxon test (not normally distributed direct comparisons), Mann-Whitney test (not normally distributed single comparisons), one-way ANOVA with Holm-Sidak's multiple comparisons test (normally distributed multiple comparisons) or Kruskal-Wallis test with Dunn's multiple comparison as a post hoc test (not normally distributed multiple comparisons) as indicated in the text. Extended Data Table 1-2 contains all applied tests and the exact $p$ values. Additionally, the Spearman correlation test was used to measure the association between cell position and branchlength in Figure $1 C$ (statistical details can be found in Extended Data Table 1-2). Numerical values are given as mean and SEM unless otherwise stated. To facilitate the interpretation of our results and the narrative flow of the paper, we followed the convention of defining $p<0.05$ as significant in the text. However, to facilitate the realistic evaluation of our data and its interpretation, we omitted 
Table 1: Intrinsic electrical properties of layer $2 a$ and layer $2 b$ neurons at p12-p14 and $>$ p30

\begin{tabular}{lllll}
\hline & $12-14 \mathrm{pd}$ & & $>30 \mathrm{pd}$ & $\mathrm{SP}$ \\
& $\mathrm{SL}$ & $\mathrm{SP}$ & $\mathrm{SL}$ & $-72.23 \pm 2.01$ \\
$\mathrm{Vm}(\mathrm{mV})$ & $-75.32 \pm 1.96$ & $-73.48 \pm 2.86$ & $-71.88 \pm 1.89$ & $235.29 \pm 51.62$ \\
$\operatorname{Rin}(\mathrm{M} \Omega)$ & $233.35 \pm 40.24$ & $147.52 \pm 27.07$ & $168.71 \pm 22.65$ & $22.53 \pm 1.48$ \\
$\mathrm{Tau}(\mathrm{ms})$ & $25.65 \pm 2.94$ & $22.98 \pm 4.24$ & $20.79 \pm 1.98$ & $114.52 \pm 10.43$ \\
$\mathrm{Cm}(\mathrm{pF})$ & $123.17 \pm 11.86$ & $156.30 \pm 10.44$ & $135.89 \pm 9.66$ & $-40.35 \pm 1.07$ \\
Threshold $(\mathrm{mV})$ & $-35.97 \pm 1.50$ & $-39.37 \pm 1.17$ & $-40.28 \pm 1.52$ & $7.96 \pm 0.77$ \\
fAHP (mV) & $7.75 \pm 1.30$ & $5.65 \pm 1.51$ & $10.83 \pm 1.59$ & $68.78(29.42-88.44)$ \\
Instant firing freq $(\mathrm{Hz})$ & $37.99(22.69-108.13)$ & $34.81(29.90-104.40)$ & $44.87(30.83-197.12)$ & Median (IQR) \\
\hline
\end{tabular}

SL refers to layer 2a neurons, SP to layer 2b neurons. See Extended Data Table 1-1, Table 1-2 for further details.

significance stars from the majority of the plots. Wherever possible, we used estimation-based statistics with meandifference plots instead (Ho et al., 2019).

\section{Results}

\section{Layer identification in postnatal development}

In acute horizontal mouse brain slices, we performed whole cell patch clamp recordings of randomly sampled excitatory neurons over the whole vertical extent of layer 2 including the layer 2/3 transition zone. Excitatory neurons were distinguished from interneurons by at least one of the three criteria: firing profile (Suzuki and Bekkers, 2010), morphology, and a negative post hoc staining for interneuron markers. During patching, neurons were filled with biocytin for later morphologic reconstructions (Fig. 1A1).

For analysis, the extent of layer 2 was delineated using a DAPI stain (Fig. 1A2). Layer 2 was divided into layer 2a (upper third) and layer $2 b$ (deep two-thirds and layer 2/3 transition zone; Choy et al., 2017; Martin-Lopez et al., 2019). We chose this division into sublayers for categorization although there is a postulated gradient of the electrophysiological and morphologic differences between deep and superficial neurons (Suzuki and Bekkers, 2011; Wiegand et al., 2011). Our rationale behind this hard segregation is the clear distribution of genetic markers in layer 2 and the necessity to find a criterion applicable to all age groups studied. All published genetic markers for superficial or layer 2a neurons seemed to display a clear and selective expression profile in the upper third of layer 2 (Diodato et al., 2016; Choy et al., 2017; Bolding et al., 2019). In addition, functional analysis of neurons expressing genetic markers of layer 2a neurons display reduced recurrent circuit incorporation (Choy et al., 2017; Bolding et al., 2019).

We confirmed the location of the aPCx by staining the LOT and layer 1a fibers with calretinin (Sarma et al., 2011; Fig. 1A3). The calretinin staining also permitted clear delineation of dendritic segments terminating in layer 1a (Fig. 1A4, see Fig. 5). 3D reconstructions using neutube (Feng et al., 2015) of layer 2a ( $n=46 / 25$ neurons/mice; Fig. 1B1) and layer $2 b(n=43 / 27$ neurons/mice; Fig. 1B2) neurons were analyzed at four time windows: right after birth (p1-p2, layer 2a: $n=6 / 4$; layer 2b: $n=12 / 6$; neurons/ mice), at the end of the first postnatal week (p6-p8, layer 2a: $n=10 / 5$; layer $2 \mathrm{~b}: n=10 / 7$; neurons/mice), during the critical period of heightened sensory synaptic and structural plasticity (p12-p14, layer 2a: $n=11 / 8$; layer 2b: $n=9$ /
8; neurons/mice; Franks Kevin and Isaacson 2005; Poo and Isaacson 2007), and after the critical period (>p30, layer 2a: $n=19 / 6$; layer 2b: $n=12 / 6$; neurons/ mice; Fig. 1B).

\section{Neurons in sublayers $2 \mathrm{a}$ and $\mathbf{2 b}$ are distinct}

Electrophysiological characterization was performed for a subset of neurons at p12-p14 (layer 2a: $n=10 / 6$; layer $2 \mathrm{~b}: n=8 / 7$; neurons, mice) and at $>$ p30 (layer 2a: $n=14 / 7$; layer 2b: $n=9 / 6$; neurons, mice). When sampling the whole extent of layer $2 \mathrm{a}$ and layer $2 \mathrm{~b}$, we did not find statistically significant electrophysiological differences between neurons in the layers $2 a$ and $2 b$ at both ages (Table 1; Extended Data Table 1-1, Table 1-2; see Discussion).

In addition to previously reported electrophysiological differences, the less complex basal dendritic tree of superficial layer 2a cells compared with deeper neurons in layer $2 \mathrm{~b}$ is a prominent distinctive feature (Bekkers and Suzuki, 2013). As basal dendritic length most likely scales with local recurrent wiring (Haberly, 1985), this is a good indicator of a layer 2 neuron's recurrent circuit incorporation, which is central to this study. After the first postnatal week, we saw differences in the architecture of the basal dendritic tree between layer $2 \mathrm{a}$ neurons and layer $2 \mathrm{~b}$ neurons. When plotting the normalized cell depth in layer 2 against the total dendritic branch length (TDBL) of each neuron's basal tree, we observed a stable superficial to deep gradient of basal dendritic tree length and complexity over postnatal development [p1-p2 $(r=0.62, p<0.01)$, p6-p8 $(r=0.68$, $p<0.01), \quad$ p12-p14 $(r=0.84, \quad p<0.0001)$, and $>$ p30 $(r=0.54, p<0.01)$; Spearman correlation test; Fig. $1 C$; Extended Data Fig. 1-1]. When using our positional grouping approach, the number of basal branches (NB) and the total basal dendritic branch length (BL) were significantly smaller in layer $2 a$ than in layer $2 \mathrm{~b}$ neurons starting in postnatal week 1 (see also Fig. 3A; NB: layer 2a vs layer 2b: p6p8: $p<0.001$, p12-p14: $p<0.0001,>$ p30: $p<0.01$; total basal dendritic length: layer $2 \mathrm{a}$ vs layer 2b: p6-p8: $p<0.01$, p12-p14: $p<0.0001$, $>$ p30: $p<0.05$; ANOVA with Holm-Sidak's multiple comparisons test). We conclude that the morphologic parameter basal dendritic length justifies the distinction between a superficial (layer $2 \mathrm{a}$ ) and deep (layer $2 \mathrm{~b}$ ) population of aPCx layer 2 neurons in our dataset.

\section{Distinct growth phases in apical and basal dendrites}

We chose a set of morphometric parameters to describe the growth of the apical (Fig. 2) and basal (Fig. 3) 
A 1 Layer 2A
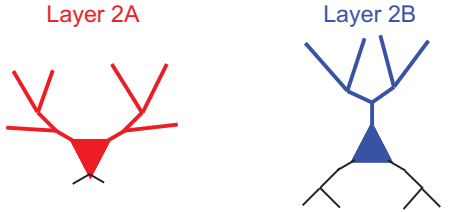

$\mathbf{A}_{2}$<smiles>[AlH2]</smiles>
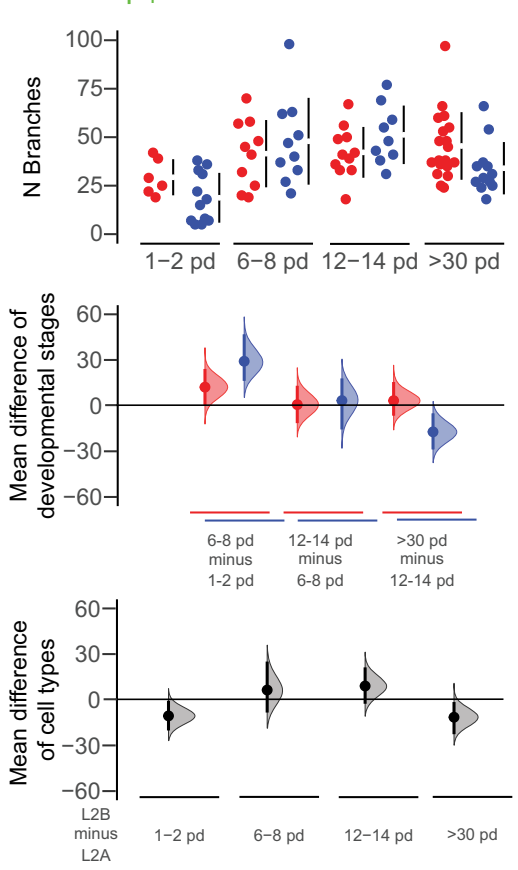

B

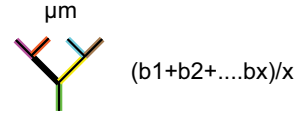

$\mathrm{A}_{3}$

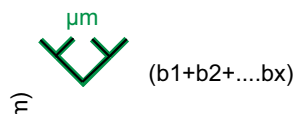

$\mathbf{A}_{4}$
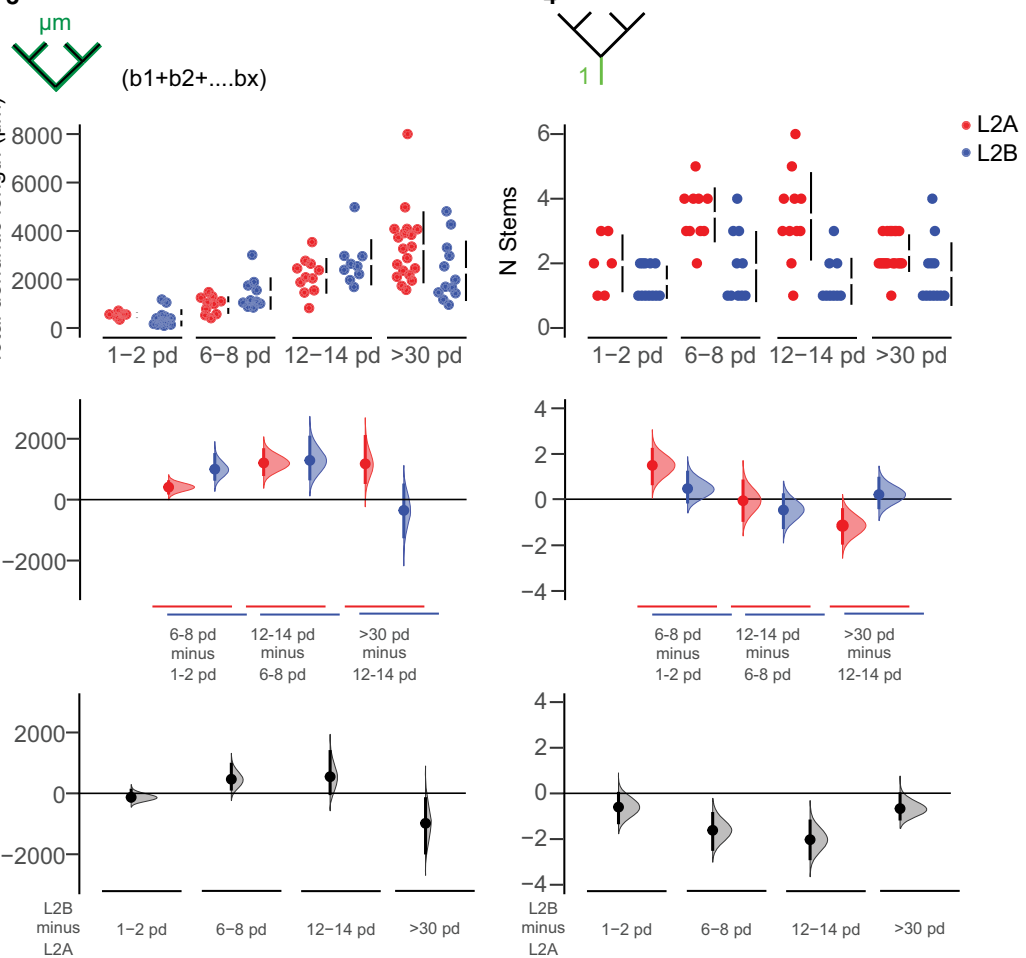

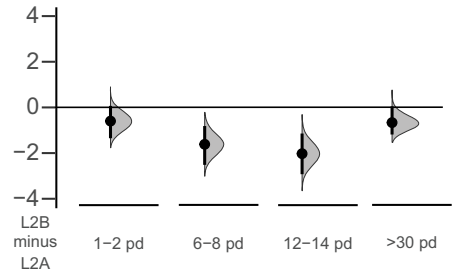

$\mathrm{C}_{1_{\text {Distribution of branches }}}$

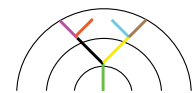

Layer $2 A$ cells

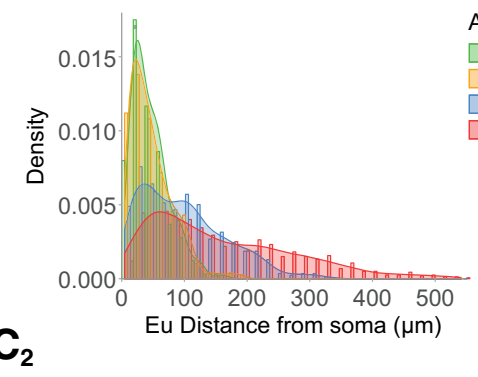

Layer 2B cells

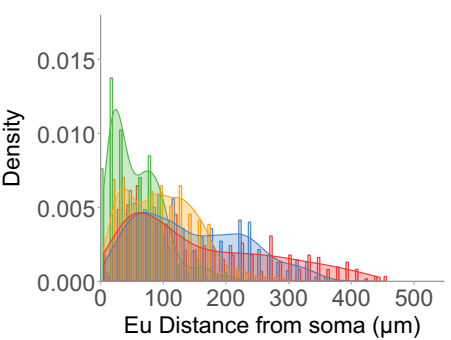


continued

Figure 2. Developmental changes in the morphology of the apical dendritic tree. A1, Visual representation of layer 2a (L2A, top, red) and layer $2 b$ ( $\mathrm{L} 2 \mathrm{~B}$, bottom, blue) neurons. Morphologic parameters are used to described growth patterns of the apical trees of these cells during development at four different ages (expressed as postnatal days, pd). Four measurements were extracted from the reconstructed cells and are displayed in Cumming estimation plots: (A2) total number of apical branches per cell; (A3) total apical dendritic length per cell; $(\boldsymbol{A} 4)$ total number of apical stems per cell; and $(\boldsymbol{B})$ average apical branch-length per cell in micrometers. The raw data are plotted on the upper axes; mean differences between developmental stages are plotted on the middle axes and mean differences between the cell types are plotted on the lower axes, as a bootstrap sampling distribution. Mean differences are depicted as dots and the $95 \%$ confidence intervals are indicated by the ends of the vertical error bars. Red dots represent layer $2 a$ neurons (L2A), blue dots represent layer 2b neurons (L2B). C , Densities of the distributions of apical branches for layer 2a (C1) and layer 2b (C2) are plotted as function of the Euclidean distance from the soma at four time windows: 1-2 pd (green), 6-8 pd (yellow), 12-14 pd (blue), and $>30 \mathrm{pd}$ (red). See Extended Data Table 1-2 for statistical details.

Figure Contributions: Laura Moreno-Velasquez performed the experiments. Laura Moreno-Velasquez, Malte Kaehne, and Friedrich W. Johenning analyzed the data.

dendritic tree: NB, TDBL, number of stems, average individual $\mathrm{BL}$, and branch density as a function of distance from the soma. Using these parameters, we defined three distinct developmental phases. In apical dendrites of both layer $2 \mathrm{a}$ and layer $2 \mathrm{~b}$ neurons, we observed the largest fractional increase in branch number in the first postnatal week (layer 2a: $75 \%$ of total increase in branch number; layer 2b: $90 \%$ of total increase in branch number; Fig. $2 A 2)$. For layer $2 b$ neurons, we observed a significant increase in apical branch number between p0-p2 and p6-p8 (layer 2b neurons: $\mathrm{p} 1-\mathrm{p} 2$ vs $\mathrm{p} 6-\mathrm{p} 8: \mathrm{p}<0.001$, p12-p14 vs >p30: $p<0.05$; ANOVA with Holm-Sidak's multiple comparisons test; Fig. 2A2). Layer 2a neurons displayed a significant addition of proximal stems in the first postnatal week only (layer 2a neurons: p1-p2 vs p6p8: $p<0.01, \mathrm{p} 12-\mathrm{p} 14$ vs $>\mathrm{p} 30: p<0.01$; ANOVA with Holm-Sidak's multiple comparisons test; Fig. 2A4). Layer $2 \mathrm{~b}$ neuron basal branches displayed a similar developmental pattern (Fig. 3A2). Here, we observed a statistically significant increase in branch number in the first postnatal week (layer 2b: $64 \%$ of total increase in branch number; Fig. 3A2). In the shorter and less complex basal tree of layer 2a neurons (Fig. $3 A$, bottom panels), significant branch addition was only evident when compared over the whole developmental period observed (layer $2 \mathrm{~b}$ neurons: $\mathrm{p} 1-\mathrm{p} 2$ vs p6-p8: $p<0.01, \mathrm{p} 12-\mathrm{p} 14$ vs $>\mathrm{p} 30: p<$ 0.05 ; layer 2a neurons: p1-p2 vs p30: $p<0.05$; ANOVA with Holm-Sidak's multiple comparisons test; Fig. 3A2).

After this initial determination of branch complexity by branch addition (developmental phase 1), dendrites grew by branch elongation (developmental phase 2). In layer 2a neurons, we observed statistically significant increases of the total apical dendritic $B \mathrm{~L}$ in the second postnatal week and between weeks 2 and 5 (Fig. 2A3). In layer 2b apical dendrites, the TDBL increased significantly in the first and second postnatal weeks (layer 2a neurons: p6-p8 vs p12$\mathrm{p} 14: p<0.05, \mathrm{p} 12-\mathrm{p} 14$ vs $>\mathrm{p} 30: p<0.05$; layer $2 \mathrm{~b}$ neurons: $\mathrm{p} 1-\mathrm{p} 2$ vs $\mathrm{p} 6-\mathrm{p} 8: \mathrm{p}<0.05$, $\mathrm{p} 6-\mathrm{p} 8$ vs $\mathrm{p} 12-\mathrm{p} 14$ : $p<0.01$; ANOVA with Holm-Sidak's multiple comparisons test; Fig. 2A3). In basal dendrites of layer $2 \mathrm{~b}$ neurons, we observed increases in TDBL in developmental phase 2 until p12-p14 (Fig. 3A3). In the shorter and less complex basal tree of layer 2a neurons, significant length growth was again only evident when comparing over the whole developmental period observed (layer 2a neurons: $\mathrm{p} 1-\mathrm{p} 2$ vs $>\mathrm{p} 30$ : $p<0.05$; layer $2 \mathrm{~b}$ neurons: $\mathrm{p0}-\mathrm{p} 2$ vs $\mathrm{p} 6-$ p8: $p<0.05, \mathrm{p} 6-\mathrm{p} 8$ vs p12-p14: $p<0.001, \mathrm{p} 12-\mathrm{p} 14$ vs $>$ p30: $p<0.001$; ANOVA with Holm-Sidak's multiple comparisons test; Fig. $3 A 3$ ).

Increases in the TDBL are a combined effect of branch addition and elongation of individual branch segments. The dichotomy between branch addition in developmental phase 1 and the length growth of existing branches in developmental phase 2 became apparent when examining the average $B L$ per neuron (Figs. $2 B, 3 B$ ). In layer 2a and layer $2 \mathrm{~b}$ neuron apical and basal branches, the average $\mathrm{BL}$ per neuron did not increase in the first postnatal week. In the second postnatal week and between weeks 2 and 5 , layer $2 \mathrm{a}$ and layer $2 \mathrm{~b}$ neurons both displayed significant increases in the average apical BL per neuron (layer 2a neurons: p6-p8 vs p12-p14: $p<0.0001, \mathrm{p} 12-$ p14 vs $>$ p30: $p<0.0001$; layer $2 b$ neurons: $p 6-p 8$ vs p12-p14: $p<0.001$, p12-p14 vs $>$ p30: $p<0.05$; ANOVA with Holm-Sidak's multiple comparisons test; Fig. 2B).

Layer $2 \mathrm{~b}$ basal branches exhibited a similar pattern, the average $\mathrm{BL}$ only increased significantly in the second postnatal week but not in the first postnatal week (Fig. $3 B$ ). In layer $2 a$ neuron basal dendrites, length increase was only significant when comparing over the whole developmental period observed (layer $2 b$ : $p 6-p 8$ vs $p 12-$ p14: $p<0.001$; layer 2a: $p 1-p 2$ vs $>$ p30: $p<0.01$; ANOVA with Holm-Sidak's multiple comparisons test; Fig. 3A3).

It is obvious from Figure $3 B$ that the relatively small length increase in layer $2 a$ neuron basal dendrites predominantly occurred in the second postnatal week and between postnatal weeks 2 and 5 . In sum, our measurements permitted us to clearly distinguish branch addition in developmental phase 1 followed by elongation of individual branch segments in developmental phase 2 for layer $2 \mathrm{a}$ and layer $2 \mathrm{~b}$ dendrites.

We identified a third developmental phase in the interval between the end of the second postnatal week (p12-p14) and the fifth postnatal week ( $>$ p30). We observed a 34\% reduction in the number of apical branches in layer $2 b$ neurons and a $33 \%$ reduction in the number of stems in layer $2 \mathrm{a}$ neurons (Fig. 2A2,A4). In layer $2 \mathrm{~b}$ neurons, this pruning was accompanied by a halt in the increase of TDBL (Fig. 2A3) despite a significant increase in the average $B L$ per neuron (Fig. $2 B$ ). This resulted in a diverging developmental trajectory of the apical dendrite between layer $2 a$ and layer $2 b$ neurons. While the TDBL was similar 
A Layer 2A

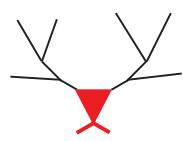

$A_{2}$
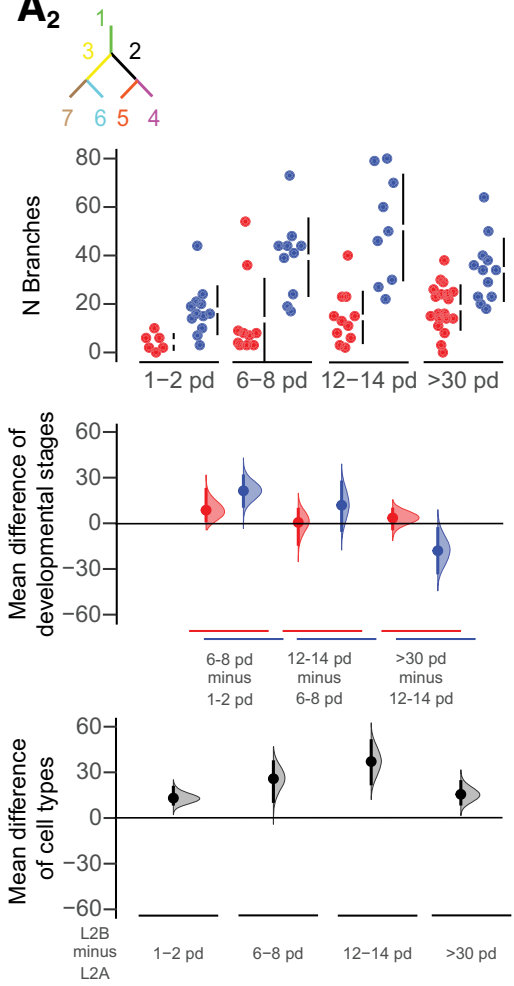

B
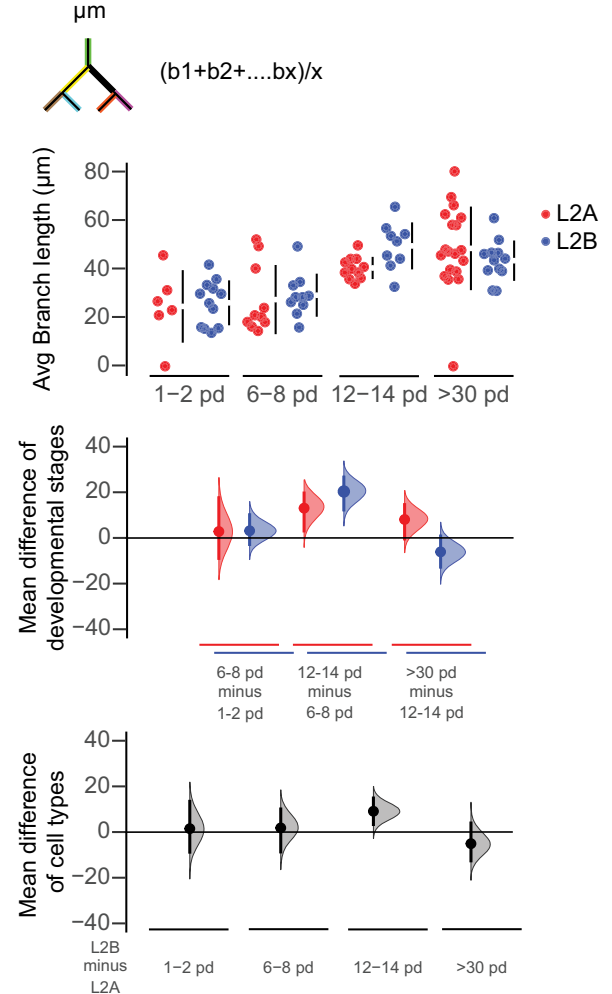

$\mathbf{A}_{3}$

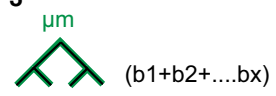

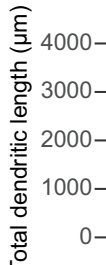

$\mathbf{A}_{4}$

$\widehat{1} \lambda$
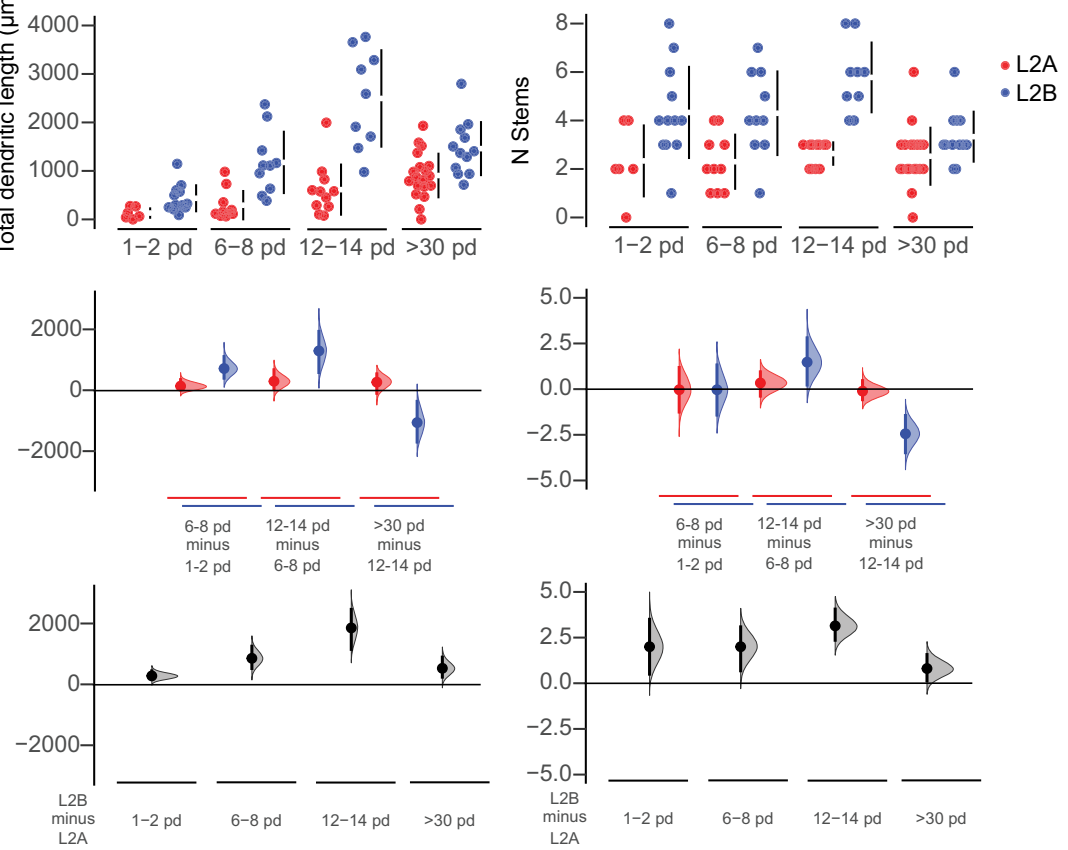

$\mathrm{C}_{1_{\text {Distribution of branches }}}$

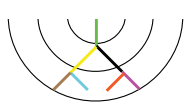

Layer $2 \mathrm{~A}$ cells

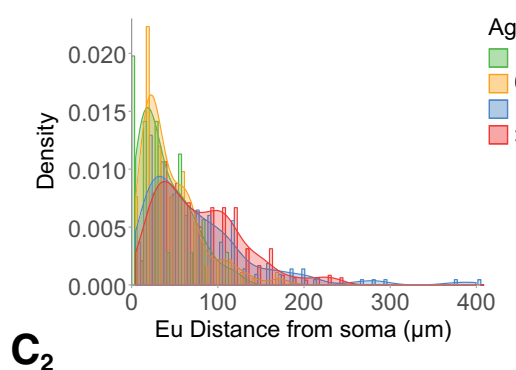

$\mathbf{C}_{2}$ Age

$1-2 \mathrm{pd}$
$6-8 \mathrm{pd}$

$12-14 \mathrm{pd}$

$>30 \mathrm{pd}$

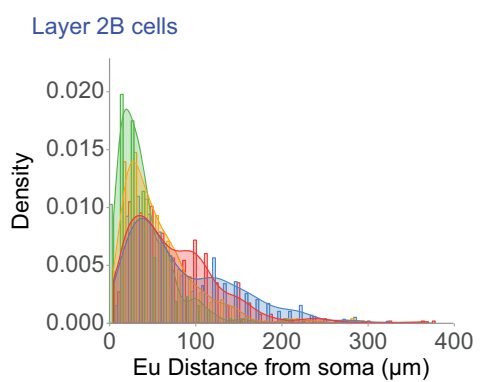


continued

Figure 3. Developmental changes in the morphology of the basal dendritic tree. A1, Visual representation of layer 2a (L2A, top, red) and layer $2 \mathrm{~b}(\mathrm{~L} 2 \mathrm{~B}$, bottom, blue) neurons. Morphologic parameters are used to described growth patterns of the basal dendritic trees of layer 2a (L2A, red) and layer 2b (L2B, blue) neurons during development at four different ages (expressed as postnatal days, pd). Four measurements were extracted directly from the reconstructed cells and are shown in Cumming estimation plots: (A2) total number of basal branches per cell; (A3) total basal dendritic length per cell in micrometers; (A4) total number of basal stems per cell; and $(B)$ average basal branch-length per cell in micrometers. The raw data are plotted on the upper axes; mean differences between developmental stages are plotted on the middle axes and mean differences between the cell types are plotted on the lower axes, as a bootstrap sampling distribution. Mean differences are depicted as dots and the $95 \%$ confidence intervals are indicated by the ends of the vertical error bars. Red dots represent layer 2a neurons (L2A), blue dots represent layer 2b neurons (L2B). C, Densities of the distributions of basal branches for layer $2 \mathrm{a}(\boldsymbol{C} 1)$ and layer $2 \mathrm{~b}(\boldsymbol{C} 2)$ are plotted as function of the euclidean distance from the soma at four time windows: p1-p2 (green), p6-p8 (yellow), p12-p14 (blue), and >p30 (red). See Extended Data Table 1-2 for statistical details.

Figure Contributions: Laura Moreno-Velasquez performed the experiments. Laura Moreno-Velasquez, Malte Kaehne, and Friedrich W. Johenning analyzed the data.

until week 2, the two different developmental patterns of layer $2 \mathrm{a}$ and layer $2 \mathrm{~b}$ neurons resulted in a significantly shorter apical dendritic tree in layer $2 b$ neurons at five weeks (layer $2 \mathrm{a}$ vs layer $2 \mathrm{~b}$ at $>$ p30: $p<0.05$; ANOVA with Holm-Sidak's multiple comparisons test; Fig. 2A3). Similar to their apical dendrites, layer $2 \mathrm{~b}$ neuron basal dendrites pruned significantly after p12-p14, both with respect to TDBL and branch number (Fig. $3 A 2, A 3$ ). Between postnatal weeks 2 and 5, we therefore defined a third developmental phase of pruning for apical and basal dendrites of layer $2 \mathrm{~b}$ neurons and apical stems of layer 2 a neurons.

To see how the three distinct developmental phases affected the spatial arrangement of branches, we plotted the distribution of branch densities as a function of distance from the soma. We observed differences between apical dendrites of layer $2 a$ and layer $2 b$ neurons during the first two postnatal weeks. In developmental phase 1, layer $2 \mathrm{a}$ neurons branched close to the soma, the distribution was single-peaked (Fig. 2C1). In contrast, layer 2b neurons also displayed a second peak of distal branching right after birth (p1-p2), and during the first developmental phase determining dendritic complexity (p6-p8; Fig. 2C2). During the second developmental phase of dendritic elongation, no branches were added in layer $2 a$ and layer $2 b$ neurons. At the same time, we observed a right shift of the peaks of apical branch density to larger distances from the soma. This indicated that length growth was not limited to dendritic tips but also affected intermediate branches. Pruning in developmental phase 3 resulted in a reduction of the second, distal peak of the layer $2 \mathrm{~b}$ neurons, approximating the apical branch distributions of layer $2 \mathrm{a}$ and layer $2 \mathrm{~b}$ neurons. The distribution of basal branch density as a function of distance from the soma was similar for both cell types, with a right shift for layer 2b neurons (Fig. 3C).

In sum, circuit-specific differences in dendritic development between layer $2 \mathrm{a}$ and layer $2 \mathrm{~b}$ neurons were observed in developmental phases one (branch addition) and three (pruning). While differences in basal branch number between layer $2 \mathrm{a}$ and layer $2 \mathrm{~b}$ neurons were determined in developmental phase 1, development of the apical dendritic tree diverged during pruning in phase 3 .

\section{Functional connectivity during early spontaneous network activity at the mesoscale population level reflects morphologic differences}

The complexity of both the apical and the basal dendritic tree is determined in the first postnatal week by branch addition (developmental phase 1). As dendritic structure and neuronal activity are interdependent, our next aim was to compare neuronal activity patterns during the first postnatal week between layer $2 \mathrm{a}$ and layer $2 \mathrm{~b}$ neurons.

We analyzed differences between the two cell types at the mesoscale population level during immature slow spontaneous network activity patterns. Similar to the somatosensory cortex, immature slow spontaneous network activity patterns in aPCx coexist with and can be triggered by sensory inputs starting at p0 (Hoffpauir et al., 2009; Leighton and Lohmann, 2016). In acute brain slices, slow spontaneous network activity is preserved as a default state of the intrinsic recurrent network (Rigas et al., 2015).

In the juvenile circuit, layer 2 a neurons are less likely to be incorporated into recurrent circuits than layer $2 \mathrm{~b}$ neurons (Suzuki and Bekkers, 2011; Wiegand et al., 2011). To date, it is unclear whether immature spontaneous network activity reflects mature connectivity patterns or acts as an unstructured global signal. Observing spontaneous network activity in the aPCx, we therefore next wanted to test whether immature spontaneous network activity early in development differentially incorporates layer $2 \mathrm{a}$ and layer $2 b$ neurons. Here, we used data from Ai95-NexCre mice as this mouse line is specific for glutamatergic neurons. To assess functional connectivity between neurons, we identified all visible neurons (4755/50/39/23 neurons/fields of view/slices/mice). Based on $\mathrm{Ca}^{2+}$-mediated changes of the fluorescence signals, events were defined as activityrelated $\mathrm{Ca}^{2+}$ signals distinguishable from baseline noise following the criteria stated in Materials and Methods. Cells displaying events were defined as active. For neurons that were active, we extracted all events of each individual neuron. For each neuron and each event, we calculated which percentage of layer 2 neurons was coactive in the same field of view at the same time. These percentage values were then averaged over all events observed in a neuron. We plotted neuronal depth in layer 2 against the average percentage of coactive neurons in the whole layer 2 field of view. Coactivity was significantly stronger in the deep layer $2 \mathrm{~b}$ neurons (deep third of layer 2 ) than in layer $2 \mathrm{a}$ neurons [superficial third of layer 2 (Fig. 4D); 1/3 layer 2 vs 3/3 layer $2: p<0.01$; unpaired $t$ test]. This was even more pronounced for the small fraction of neurons recorded in layer $1 \mathrm{~b}$ and in layer 3 (Fig. 4C). The degree of coactivity is an 

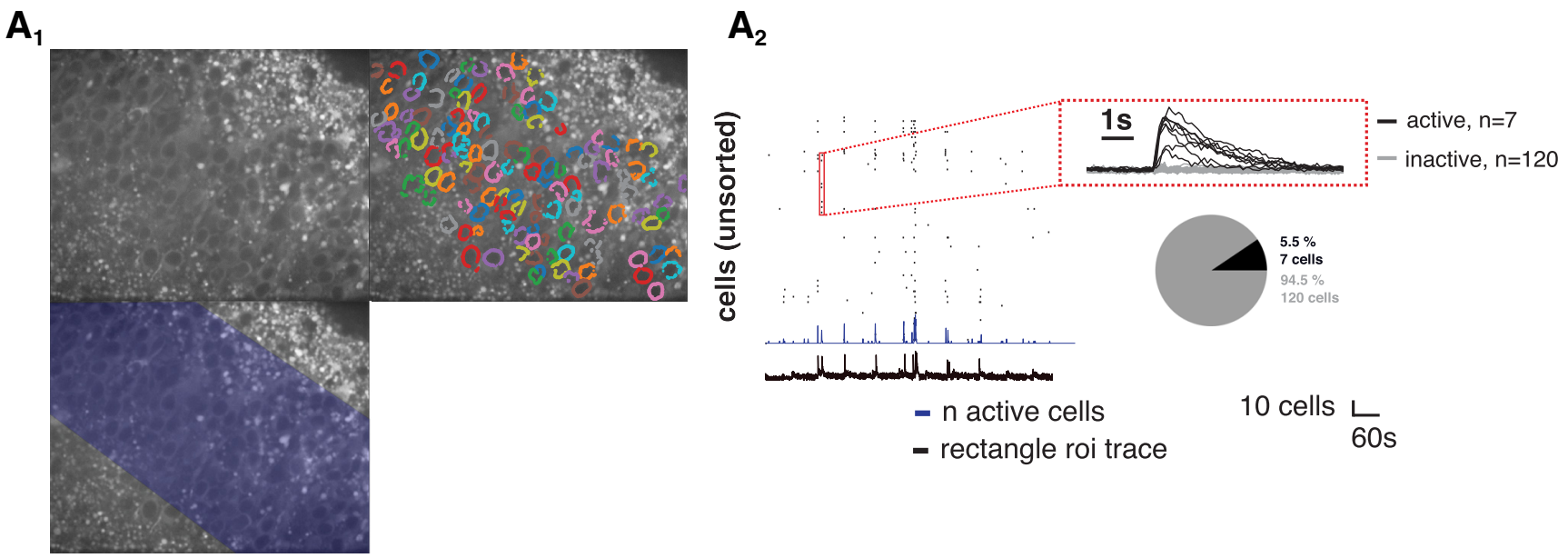

B

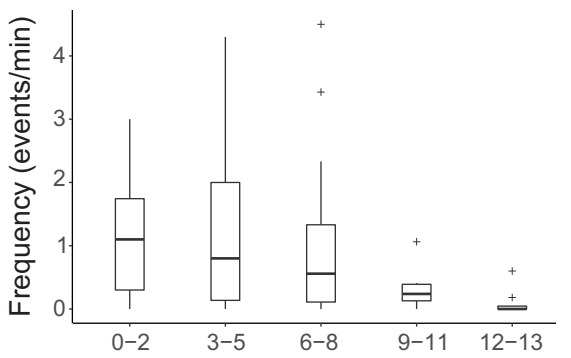

Age group (pd)
C

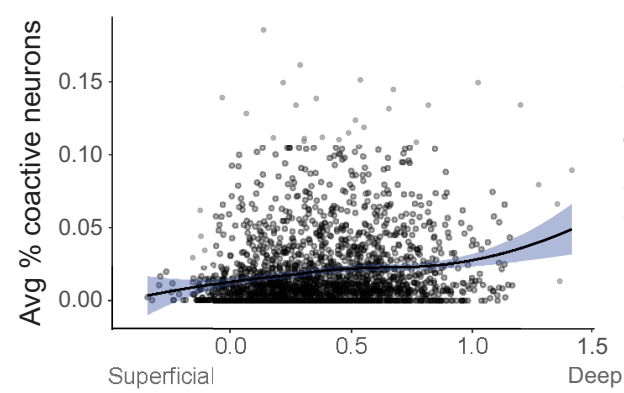

Position in Layer 2
D

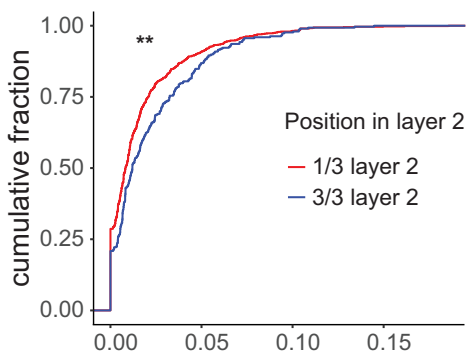

Avg \% coactive neurons

Figure 4. Comparison of spontaneous network activity in layer $2 \mathrm{a}$ and layer $2 \mathrm{~b}$ neurons. A1, Example of a baseline GCaMP-fluorescence image from an Ai95-NexCre mice. The field of view covers layer 2 in aPCx. Top right, Detected cells in layer 2 . Bottom, Rectangular ROI defined by the upper and lower boundaries of layer 2 for detecting global activity. A2, Corresponding traces from the global events measured from the rectangular ROI (fluorescence: black trace, bottom) and the active cells (raster plot, blue trace for number of active cells, red inset for fluorescent traces from individual neurons in raster plot). The proportion of active versus inactive cells is indicated by pie chart for one event. $\boldsymbol{B}$. Frequency of spontaneous events per minute measured at five different age groups (expressed as postnatal days, pd; $n=70 / 59 / 36$ fields of view/slices/mice). $\boldsymbol{C}$, For each active neuron, the average percentage of coactive neurons (based on the total number of neurons in the field of view) was plotted against the position of the active neuron in layer 2 in the aPCx (data are fitted with a local polynomial regression and pooled from the first postnatal week). $\boldsymbol{D}$, Layer 2 was divided in three parts. Cumulative distribution of the average percentage of coactive neurons from $\boldsymbol{C}$ was plotted for the superficial third (red, $1 / 3$ layer 2, corresponds to layer 2 a neurons) and the deep third (blue, $3 / 3$ layer 2 , corresponds to layer $2 b$ neurons); $* * p<0.01$. See Extended Data Table 1-2 for statistical details.

Figure Contributions: Stephen Lenzi performed the experiments and analyzed the data.

indirect measure of functional connectivity. Already during the first postnatal week, we observed higher local functional connectivity in layer $2 \mathrm{~b}$ than in layer $2 \mathrm{a}$ neurons. Morphologically, this scales with the more complex basal dendritic tree receiving more recurrent inputs (Haberly, 1985). We conclude that divergence of the basal dendritic tree complexity between layer $2 \mathrm{a}$ and layer $2 \mathrm{~b}$ neurons is already evident in the first postnatal week and reflected by differences in functional connectivity during early spontaneous network activity.

\section{Pruning in layer 1a during the early critical period of sensory plasticity}

Next, we wanted to further understand differences in the developmental pattern of the apical dendritic tree.
Here, the most pronounced differences occurred between the end of postnatal week 2 and the fifth postnatal week (developmental phase 3). In this period, we observed selective pruning of layer $2 \mathrm{~b}$ neuron apical dendrites, which did not occur in layer 2a neurons (see Fig. 2).

The distinct organization of synaptic inputs to aPCx apical dendrites enabled us to relate pruning to specific circuits by grouping dendritic branches based on their position (layer 1a for branches receiving sensory inputs and layer $1 \mathrm{~b} / 2$ for recurrent inputs). We therefore analyzed the growth and pruning patterns of apical dendrites with respect to the synaptic input layer the segments terminated in. Apical branches were categorized as branches terminating in layer 2 , layer $1 \mathrm{~b}$ (both recurrent), and layer $1 \mathrm{a}$ (sensory) for both cell types (Fig. 5A). Calretinin staining was used as a marker to delineate layer 1a (Fig. 1A3,A4). 
A

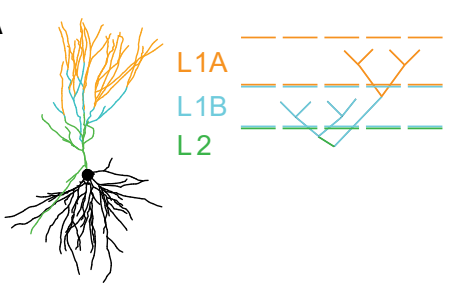

$\mathrm{B}_{1}$
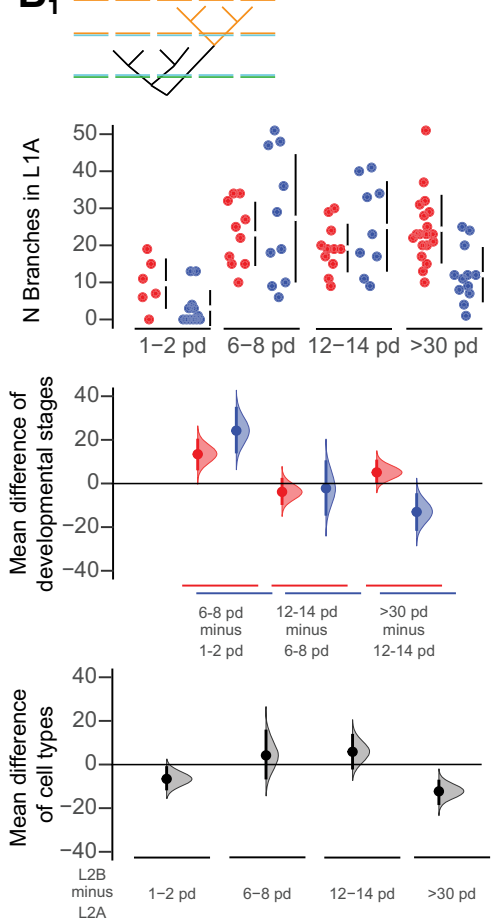

$\mathrm{C}_{1}$

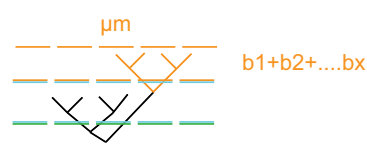

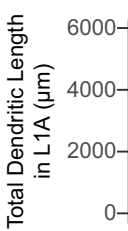
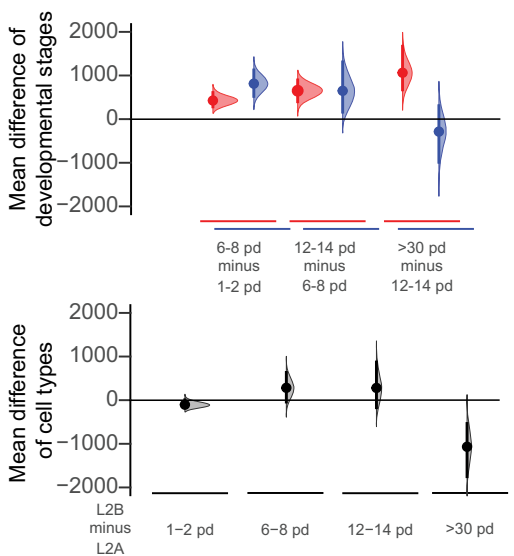
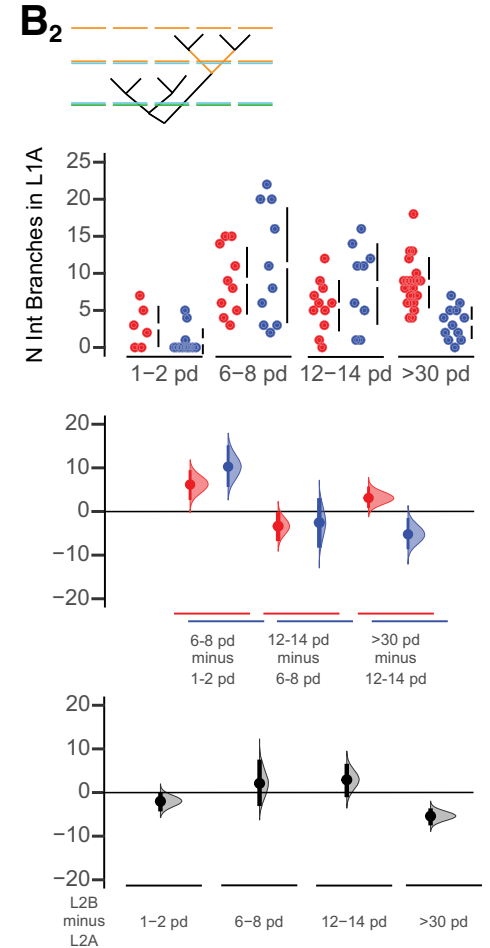

$\mathrm{C}_{2}$
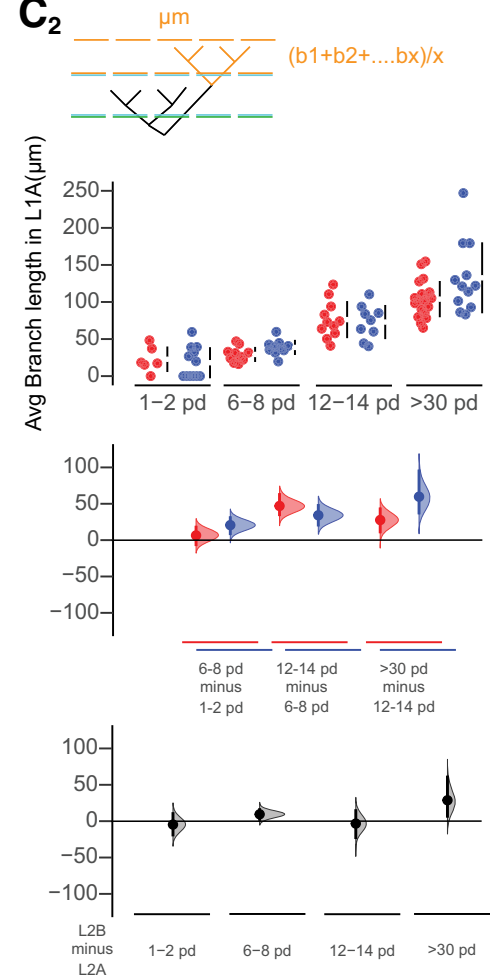
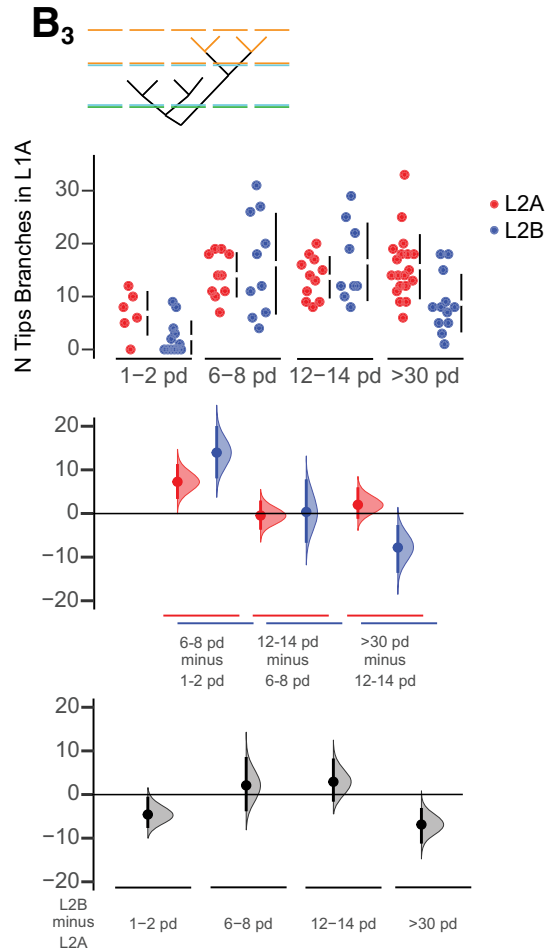

D1 Distribution of branches

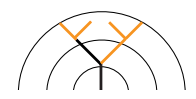

Layer 2A cells
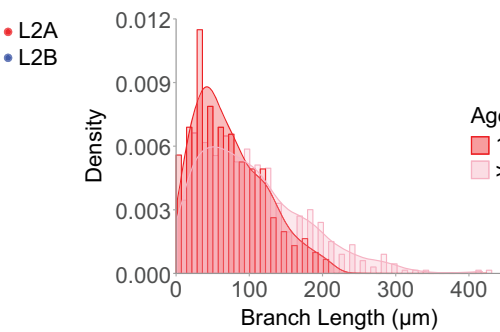

$\mathbf{D}_{2}$

Layer 2B cells

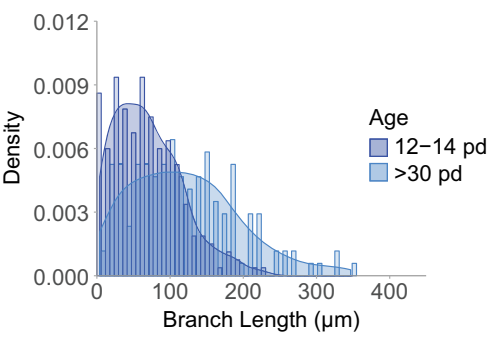


continued

Figure 5. Differences in growth pattern of apical dendrites in response to layer-specific synaptic inputs. $\boldsymbol{A}$, Example of a reconstructed cell shows the classification of the apical dendrites into three categories: branches terminating in layer 2 ( $\mathrm{L} 2$, green), layer 1b (L1B cyan), and layer 1a (L1A, orange). B, Growth patterns of the apical branches terminating in layer 1a are shown in Cumming estimation plots, including total NB per cell (B1), total number of intermediate branches per cell (B2), total number of tips per cell (B3), total dendritic length per cell (C1), and average BL per cell (C2). The raw data are plotted on the upper axes; mean differences between developmental stages (expressed as postnatal days, pd) are plotted on the middle axes and mean differences between the cell types are plotted on the lower axes, as a bootstrap sampling distribution. Mean differences are depicted as dots and the 95\% confidence intervals are indicated by the ends of the vertical error bars. Red dots represent layer $2 a$ neurons (L2A), blue dots represent layer 2b neurons (L2B). D, Densities of the distributions of the layer 1a branches for layer 2a (D1) and layer 2b (D2) neurons plotted as function of BL at two time windows: $12-14$ pd (red and blue, respectively) and $>30$ pd (pink and light blue, respectively). See Extended Data Table 1-2 for statistical details and Extended Data Figure 5-1 for growth patterns in layer $1 \mathrm{~b}$ and layer 2.

Figure Contributions: Laura Moreno-Velasquez performed the experiments. Laura Moreno-Velasquez, Malte Kaehne, and Friedrich W. Johenning analyzed the data.

While basal branches of layer $2 b$ neurons displayed clear pruning (see Fig. 3), reduction of the proximal apical branches only receiving recurrent input in layers $1 \mathrm{~b}$ and 2 did not reach statistical significance (Kruskal-Wallis test with Dunn's multiple comparison; Extended Data Fig. $5-1)$. Only in layer $2 b$ neurons, distal branches that constituted sensory layer 1a circuits pruned significantly between p12-p14 and >p30 (layer 2b neurons: p12-p14 vs p30: $p<0.05$; ANOVA with Holm-Sidak's multiple comparisons test; Fig. 5B1). Here, pruning resulted both in a significant reduction of the number of layer 1a intermediate branches and tips (intermediate branches: layer $2 \mathrm{~b}$ neurons $\mathrm{p} 12-\mathrm{p} 14$ vs $>$ p30: $p<0.05$; tips: layer $2 \mathrm{~b}$ neurons p12-p14 vs $>$ p30: $p<0.05$; ANOVA with HolmSidak's multiple comparisons test; Fig. 5B2,B3). Branch numbers in superficial layer $2 a$ neurons remained stable over this developmental period (Fig. 5B). The reduction in branch number was accompanied by a significantly shorter layer $1 a$ total dendritic length when comparing layer $2 a$ and layer $2 \mathrm{~b}$ neurons (layer $2 \mathrm{a}$ vs layer $2 \mathrm{~b}$ neurons at $>$ p30: $p<0.001$; ANOVA with Holm-Sidak's multiple comparisons test; Fig. 5C1). Pruning was therefore limited to a specific compartment in a subpopulation of neurons.

To further understand circuit and cell-type-specific pruning of the apical dendrite, we compared the average individual $\mathrm{BL}$ per neuron in layer 1a of layer 2a and layer $2 \mathrm{~b}$ neurons between postnatal weeks 2 and 5 . We observed significant branch elongation of individual layer 1a branches for both cell types (Fig. 5C2). When comparing layer $2 \mathrm{a}$ and layer $2 \mathrm{~b}$ neurons, the average layer $1 \mathrm{a} B \mathrm{BL}$ per neuron was similar after the second postnatal week. However, after the pruning phase, layer $1 \mathrm{a}$ branches in layer $2 b$ neurons were significantly longer ( $p 6-p 8$ vs $p 12-$ p14: layer 2a neurons: $p<0.0001$, layer $2 b$ neurons: $p<0.05$; $12-\mathrm{p} 14$ to $<\mathrm{p} 30$ : layer $2 \mathrm{a}$ neurons: $p<0.01$ layer $2 b$ neurons: $p<0.001$; layer $2 a$ neurons vs layer $2 b$ neurons at $>$ p30: $p<0.05$; ANOVA with Holm-Sidak's multiple comparisons test; Fig. 5C2). When analyzing the distribution change of average BLs during the pruning phase, we saw a shift toward longer 1a branches in both cell types, with a more pronounced shift in $2 b$ neurons (Fig. 5D1,D2). In layer 2a neurons, we did not observe layer 1 a branch loss. Here, the length increase could be explained by branch elongation, which is a consequence of cortical growth. However, when interpreting the distribution shift in layer $2 \mathrm{~b}$ neurons, we had to consider the pruning-related branch loss. The stronger right shift of the distribution toward longer branches in comparison to layer 2a neurons was accompanied by a significant decrease in branch number. This implied that pruning of sensory layer $1 \mathrm{a}$ branches in layer $2 \mathrm{~b}$ neurons predominantly affected short branches or that surviving branches underwent enhanced length growth.

\section{NMDA-spikes are more pronounced in layer 1a dendrites of layer $\mathbf{2 b}$ neurons}

We next investigated the connection between the preferential pruning of short layer 1a branches and the recent discovery of supralinear dendritic integration of sensory inputs in layer 1a branches of PCx layer $2 \mathrm{~b}$ neurons. As was recently shown in rat $\mathrm{aPCx}$ layer $2 \mathrm{~b}$ neurons, clustering of synaptic inputs on the same branch as opposed to distributed input to the entire dendritic arbor significantly modified the stimulus-response behavior. Clustered (same branch) input triggered supralinear stimulus-response behavior defined as NMDAR-mediated $\mathrm{Ca}^{2+}$ spikes (NMDA-spikes). Supralinear stimulus-response behavior resulted in large local dendritic depolarization and associated $\mathrm{Ca}^{2+}$ influx. Distributed inputs of similar strength evoked substantially lower levels of dendritic depolarization (Kumar et al., 2018).

We would like to relate these findings to our morphologic data. Our experiments demonstrated that during postnatal development, pruning of the apical dendrites was mainly based on the loss of short layer 1a branches (Fig. 5C,D). We hypothesize that the selection bias toward longer branch segments in the sensory synaptic input space of layer $2 \mathrm{~b}$ neurons could be a developmental mechanism supported by supralinear stimulus-response behavior. A selection bias for long branches would optimize the efficiency of the neuronal input-output function by promoting the growth and survival of branches with a higher probability of receiving clustered (same branch) input.

So far, NMDAR-dependent supralinear stimulus-response behavior has only been demonstrated in the aPCx of rats older than four weeks (Kumar et al., 2018). Here, we hypothesize that supralinear stimulus-response behavior may play a role in the developmental branch selection observed in layer $1 a$ dendrites of layer $2 b$ neurons during the critical period ( $p 12-p 14$ to $>p 30$ ). To substantiate our hypothesis, we first wanted to test whether supralinear stimulus-response behavior also occurs in in 
distal apical layer $1 \mathrm{a}$ dendrites of layer $2 \mathrm{~b}$ neurons during postnatal week 3 . We chose a developmental interval between our morphologic observation points (p12-p14 and $>$ p30) as we propose that this is the time interval where supralinear stimulus-response behavior has an impact on the pruning mechanism. In contrast to layer $2 b$ neurons, layer 2a neurons did not show pruning of short branches but equally distributed growth. If supralinear dendritic stimulus-response behavior enhanced selective pruning in distal apical dendrites of layer $2 b$ neurons, another experimentally testable prediction would be an absence of supralinear stimulus-response behavior in layer $2 \mathrm{a}$ neurons.

We therefore performed combined somatic whole cell patch clamp recordings and two-photon $\mathrm{Ca}^{2+}$ imaging in apical layer 1 a branches of layer $2 \mathrm{a}(n=5 / 5 / 5$, neurons/slices/mice) and layer $2 \mathrm{~b}(n=5 / 5 / 5$, neurons/slices/mice) neurons. Synaptic stimulation was achieved by focal electrical stimulation with $\theta$-glass electrodes (Fig. 6A4,B4). Using $\mathrm{Ca}^{2+}$ imaging, we first identified a focal stimulation spot. Stimulation strength was then linearly increased. When plotting the area under the curve (AUC) of the EPSP in layer $2 b$ neurons, we observed a distinct supralinear increase at specific stimulation strengths, which was abolished after washing in of the NMDAR-antagonist APV (Fig. 6A3). In addition, this increase displayed the typical shape of an NMDA-spike (Fig. 6A1). We performed $\mathrm{Ca}^{2+}$ imaging in parallel and could further observe that the nonlinear enhancement of the EPSP AUC was accompanied by a branch-specific increase in spatial spread and amplitude of the $\mathrm{Ca}^{2+}$ transient (Fig. 6A5). These hallmarks of supralinear dendritic NMDA-spikes were not observed in layer 2a neurons (Fig. 6B). Next, we plotted the AUC and the amplitude of layer $2 a$ and layer $2 b$ neuron EPSPs before and after wash-in of APV. We observed a significant decrease of both parameters in layer $2 b$ neurons. In contrast, NMDAR-block by APV did not affect AUCs and amplitudes of EPSPs in layer 2a neurons (layer 2b, AUC: pre vs post: $p<0.05$; layer $2 \mathrm{a}$, pre vs post: $p=0.18$; amplitude: layer $2 \mathrm{a}$, pre vs post: $p<0.05$; layer $2 \mathrm{~b}$, pre vs post: $p=0.7$; paired $t$ test; Fig. $6 C 1, C 2)$. At the stimulation site, the stimulation-evoked $\mathrm{Ca}^{2+}$ signal of both layer $2 \mathrm{a}$ and layer $2 \mathrm{~b}$ neurons was significantly reduced by APV (layer $2 \mathrm{~b}$, pre vs post: $p<0.01$; layer $2 \mathrm{a}$, pre vs post: $p<0.05$; ratio paired $t$ test; Fig. $6 D$ ). Under NMDAR-block by APV, the AUC and the amplitude of the EPSP constitute a readout of the AMPA-type glutamate receptormediated depolarization. Consequently, the absolute value of the AUC and the amplitude in APV reflected the synaptic input strength of our stimulation. These values were not significantly different between layer $2 \mathrm{a}$ and layer $2 b$ neurons in our sample, indicating comparable stimulation strength for both cell types (AUC: layer 2a post vs layer $2 \mathrm{~b}$ post: $p=0.92$; amplitude: layer $2 \mathrm{a}$ post vs layer $2 \mathrm{~b}$ post: $p=0.25$, $t$ test; Fig. $6 C$ ).

We conclude that, in contrast to layer $2 b$ neurons, layer 2a neurons do not display supralinear stimulus-response behavior in layer 1a at comparable input strengths. In layer $2 \mathrm{~b}$ neurons, supralinear stimulus-response behavior can be observed during the developmental period of dendritic pruning of short dendritic segments. In layer 2a neurons, we neither observe pruning nor supralinear stimulus-response behavior in the same developmental period.

We next applied computational modeling to test whether the probability of supralinear integration by clustered inputs could indeed scale with BL over the range of BLs measured in this study. As a quantitative framework, we used the range of average BLs we observed at the beginning of the critical period (p12-p14; 40-110 $\mu \mathrm{m})$. The average TDBL $(1800 \mu \mathrm{m})$ was set constant for all model neurons. We estimated the layer $1 \mathrm{a}$ input density based on a recent comprehensive quantitative description of mouse piriform cortex (Srinivasan and Stevens, 2018). Based on Srinivasan and Stevens, we extrapolated that the whole population of 3700 bulbar glomeruli makes 2366 synapses with each individual neuron. Consequently, one glomerulus makes on average 0.64 synapses with each neuron. A total of 109 coincidently activated glomeruli would therefore activate 70 synapses on a layer $2 \mathrm{~b}$ neuron, which we defined as the upper limit of coincident inputs. Figure $7 B$ displays the results of our calculation. We plotted the probability of dendritic NMDA-spikes evoked by clustering of $>10$ synapses on an individual branch as a function of the average BL per neuron. This was repeated for different numbers of coincident synaptic inputs (for details, see Materials and Methods). The dendritic spike probability increased with increasing BL. Within our parameter space, clustering probability and the resulting dendritic spiking increased from close to $0 \%$ to up to $17 \%$ when comparing the shortest and longest average BL we observed in our dataset. Quantitatively, our results suggest that, during our developmental time window, dendritic NMDA-spikes are more likely to occur in long dendritic segments than short ones. It is therefore plausible that the supralinear stimulus-response behavior observed exclusively in layer $2 b$ neurons could constitute a selection mechanism optimizing the efficiency of the neuronal inputoutput function. This would occur by promoting the growth and survival of branches with a higher probability of receiving clustered (same branch) input.

\section{Discussion}

Here, we performed a morphometric and functional analysis of postnatal dendritic development in aPCx sensory and recurrent circuits. We compared developmental patterns of excitatory neurons in the sublayers $2 a$ and $2 b$. The two neighboring sublayers differ with respect to sensory and recurrent wiring. We could identify a timeline defined by three developmental phases: (1) branch addition (developmental phase 1, postnatal week 1); (2) branch elongation (developmental phase 2, postnatal week 2); and (3) branch pruning (developmental phase 3, postnatal weeks 3-5). We discovered circuit and sublayer-specific differences in dendritic development in developmental phases 1 and 3. In developmental phase 1, layer 2a neuron basal dendrites incorporated in recurrent circuits branched significantly less than layer $2 \mathrm{~b}$ neuron basal dendrites. This was accompanied by lower functional connectivity of layer $2 a$ neurons during spontaneous immature network activity. In developmental phase 3, 
$A_{1}$

$\mathrm{A}_{2}$

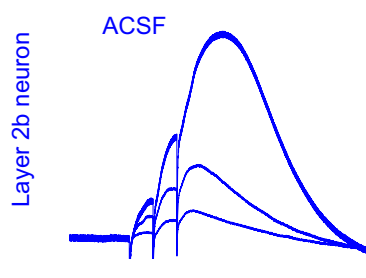

D-APV

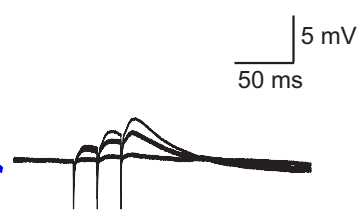

$A_{5}$
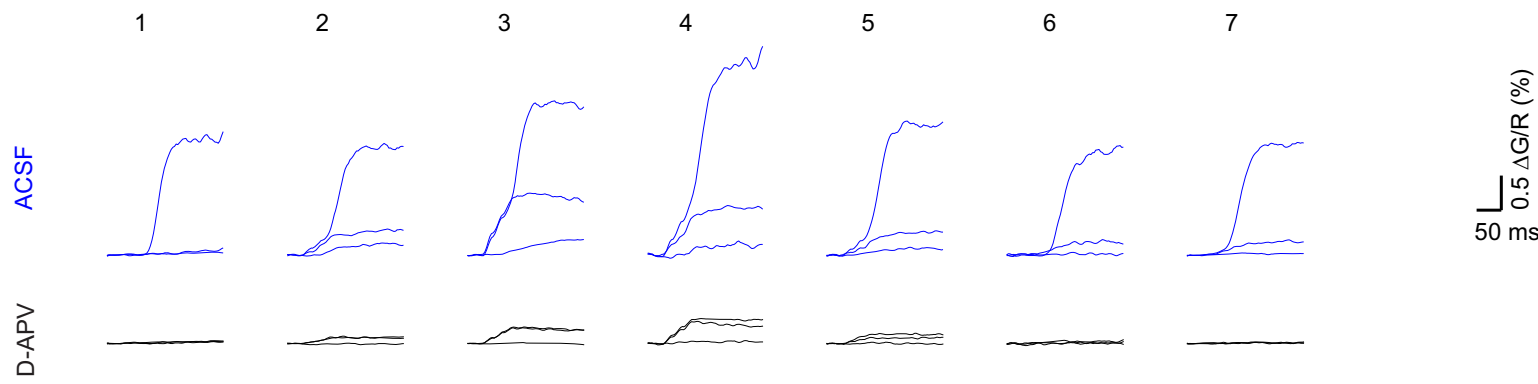

$B_{1}$

$\mathrm{B}_{2}$

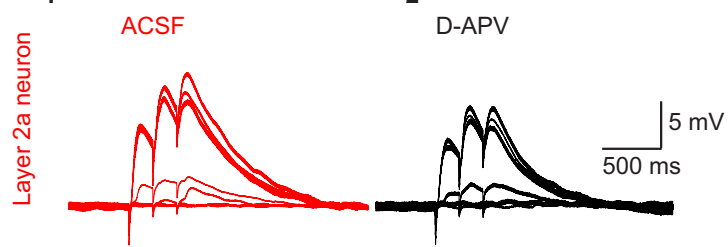

$\mathbf{B}_{5}$
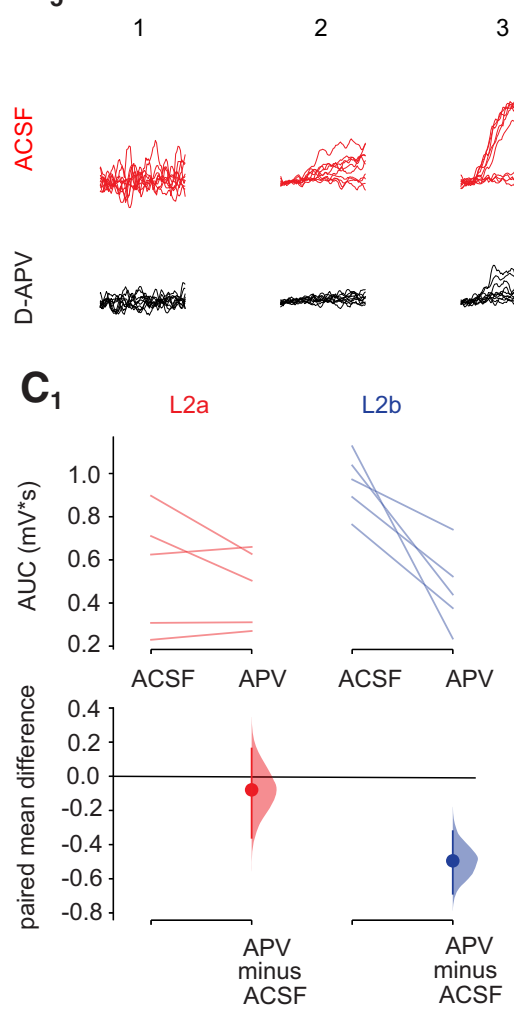

$\mathrm{C}_{2}$
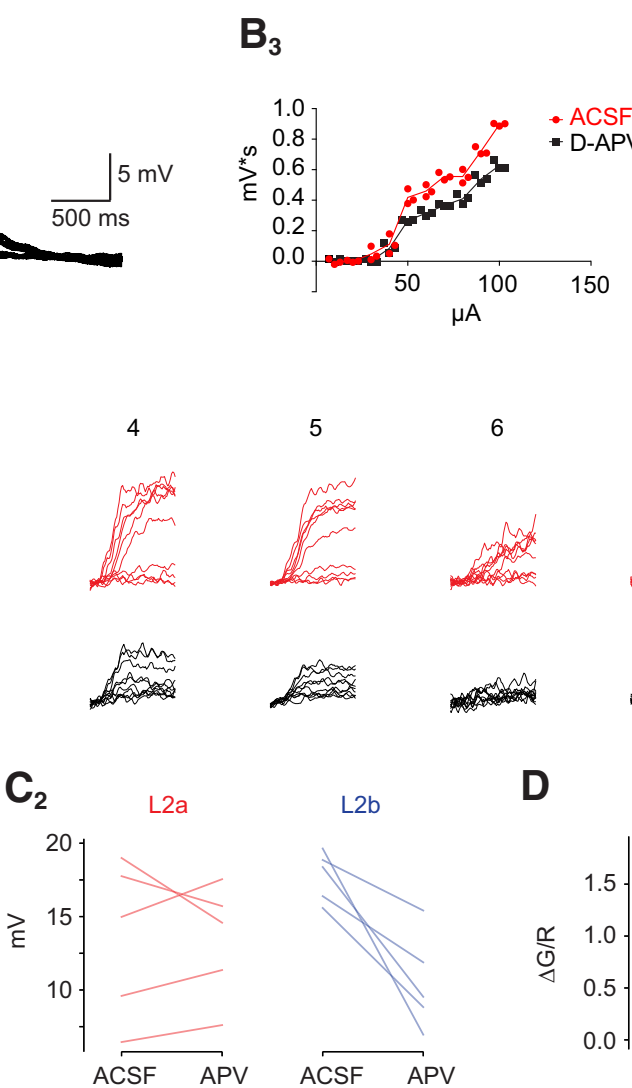

$\mathrm{B}_{4}$

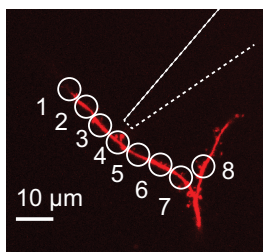

5

6

7

8

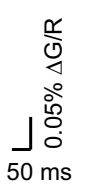

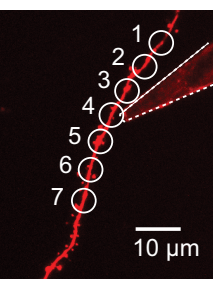

$\mathbf{A}_{4}$

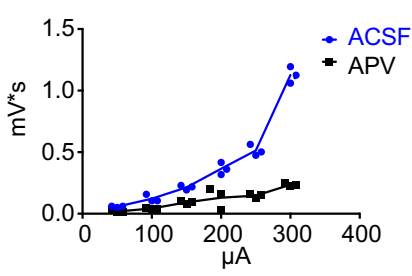

$\mu \mathrm{m}$

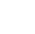


continued

Figure 6. Dendritic NMDA-spikes can only be observed in layer $2 b$ neurons. Representative layer $2 b$ neuron $(\boldsymbol{A})$ and representative layer 2a neuron $(\boldsymbol{B})$. $\boldsymbol{A} \mathbf{1}, \boldsymbol{B 1}$, Overlay of electric responses measured the soma on electrical stimulation with linear strength increase in layer 1a. A2, B2, Response to same stimulus after application of APV. A3, B3, Plot of stimulation strength against AUC from the same cell. $\boldsymbol{A 4}, \boldsymbol{B} \mathbf{4}$, Imaged dendritic branch and position of the stimulation electrode. A5, $\boldsymbol{B 5}$, Fluorescence traces of $\mathrm{Ca} 2+$ responses at ROls outlined in A4/B4 under baseline conditions (upper rows, blue and red) and after application of APV (lower rows, black). Increasing stimulation strengths are overlaid. $\boldsymbol{C}, \boldsymbol{D}$, Cell-type-specific changes to NMDAR-block with APV are plotted as Cumming estimation plots. The raw data are plotted on the upper axes, each pair is connected by a line; each mean difference is plotted on the lower axes as a bootstrap sampling distribution. Mean differences are depicted as dots and the 95\% confidence intervals are indicated by the ends of the vertical error bars. Red dots represent layer $2 a$ neurons ( $22 a)$, blue dots represent layer $2 b$ neurons (L2b). C1, AUC before and after application of APV in layer 2a neurons (L2a, red) and layer 2b neurons (L2b, blue). C2, EPSP amplitude before and after application of APV in layer $2 a$ neurons (red) and layer $2 b$ neurons (blue). $\boldsymbol{D}$, Fluorescent Ca ${ }^{2+}$ response before and after application of APV in layer $2 a$ neurons (red) and layer $2 b$ neurons (blue). Please note that we measured $\mathrm{Ca}^{2+}$ responses in five layer $2 \mathrm{~b}$ neurons; however, the datapoints of two measurements were very close and occlude each other. See Extended Data Table 1-2 for statistical details.

Figure Contributions: Hung Lo performed the experiments and analyzed the data.

pruning of apical dendrites receiving layer 1a sensory inputs was only observed in layer $2 \mathrm{~b}$ neurons, but not in layer $2 \mathrm{a}$ neurons. Pruning was clearly biased toward shorter dendritic branches. Using electrophysiology, $\mathrm{Ca}^{2+}$ imaging and modeling, we demonstrated how NMDAR-dependent supralinear stimulus-response behavior during phase 3 could support the survival and growth of long dendritic branches. Nonlinear dendritic properties could therefore be involved in dendritic development.

\section{Distinction between layer $2 \mathrm{a}$ and layer $\mathbf{2 b}$ neurons}

Distinguishing principle cell types in layer 2 poses a "lumping versus splitting" problem. Our hard segregation into layer $2 \mathrm{a}$ and layer $2 \mathrm{~b}$ neurons based on location on the vertical axis qualifies as a "lumping" approach. Along the vertical axis of layer 2, we can distinguish at least two different types of principal cells based on their position, connectivity and morphology. Based on this distinction, principal neurons in layer 2 have been hypothesized to represent two parallel streams of olfactory information processing. Superficial neurons receive predominantly sensory inputs, whereas deep neurons receive both sensory and recurrent inputs (Suzuki and Bekkers, 2011; Wiegand et al., 2011). When segregating layers $2 a$ and $2 b$ as done here, this dichotomy is complicated by the observation that there seems to be a vertical gradient between so-called semilunar cells with no basal dendrites and superficial pyramidal cells with elaborate basal dendrites (Bekkers and Suzuki, 2013). Our detailed morphologic reconstructions and analysis support the idea of a continuum between these differentially wired types of neurons. We observed short or absent basal dendrites in layer 2a and increasing basal dendritic complexity in layer $2 \mathrm{~b}$. It is established that basal dendrites primarily receive local recurrent connections (Luskin and Price, 1983; Haberly, 1985). Therefore, basal dendritic complexity is a robust criterion for distinguishing between neuronal subtypes receiving different amounts of recurrent input. We were

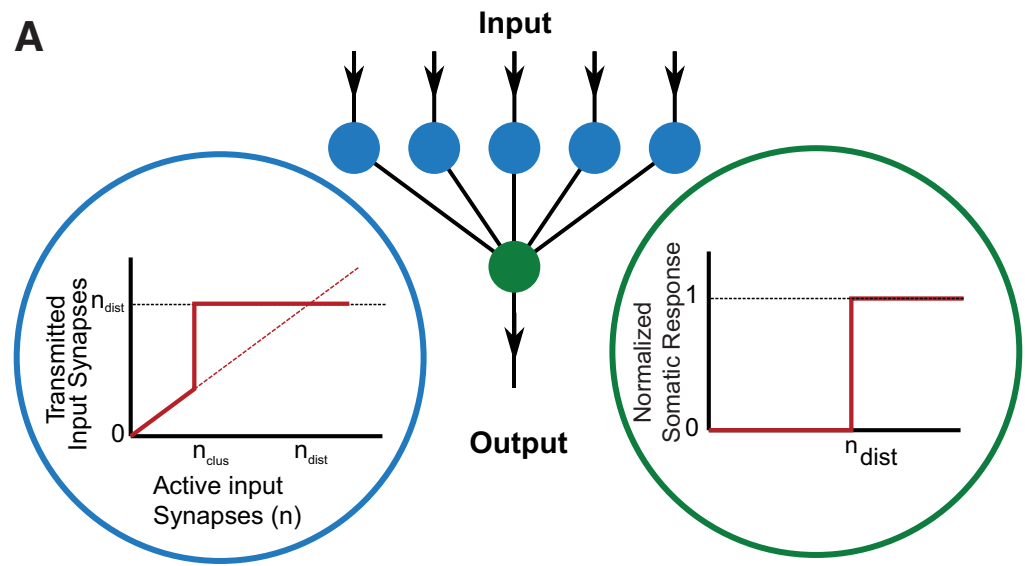

B

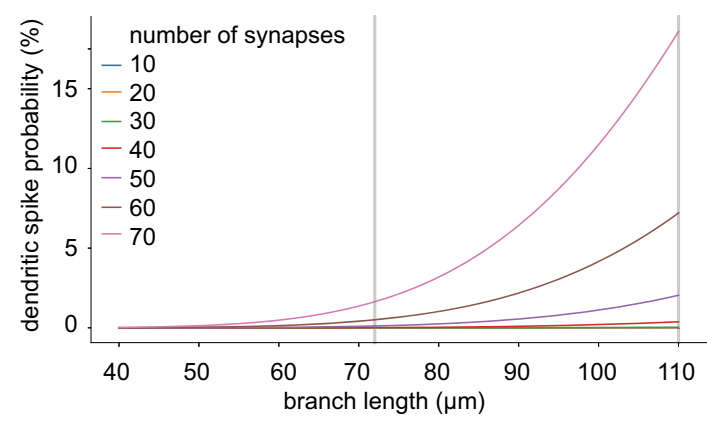

Figure 7. A, Schematic neuron model as two-layered neural network, consisting of a dendritic activation layer (blue circles) and a somatic activation layer (green circles). The activation functions for the respective layers are shown in the insets. The activation of the soma is modeled as simple step function (if the input surpasses a certain threshold, the soma responds). The activation of every separate dendrite branch is modeled as an overlay of a linear response and a (nonlinear) step function, to mimic the excitability of dendritic branches. If a dendritic branch is excited (due to a large enough number of same branch active input synapses), it transmits, triggering a somatic response. $\boldsymbol{B}$, The response probability increases as function of the BL, an effect that becomes more apparent for different increasing numbers of active input synapses $\mathrm{S}$.

Figure Contributions: Malte Kaehne performed all computations. 
therefore able to differentiate between neurons in layer $2 \mathrm{a}$ and layer $2 \mathrm{~b}$ on a vertical axis. This is important, as the intrinsic properties we observed along the vertical axis of layer 2 were more homogenous, which is different from previously published results in mice (Suzuki and Bekkers, 2006, 2011) and rats (Wiegand et al., 2011). Of all our age groups, our p12-p14 data most closely match the p13p30 observation window used for the initial description of electrophysiological differences between superficial and deep layer 2 neurons in a mouse strain closely related to ours (Suzuki and Bekkers, 2006). While we found a similar trend regarding the difference in IR and AP threshold, our cell population segregated less clearly regarding the initial firing frequency. In addition, superficial neurons in our dataset were as hyperpolarized as deep neurons, whereas more depolarized superficial layer 2 neurons were recorded in earlier studies (Table 1; Extended Data Table 11). We can list a number of reasons that could underly the observed differences: differences in sampling (covering the whole extent of layer 2 in our case rather than focusing on the lower layer 2/3 and upper border as has been done previously, a more stringent definition of the deep layer 2 border that minimizes layer three neurons displaying more pronounced bursting); different positions on the anteriorposterior axis; holding potential (as opposed to earlier studies, our layer $2 \mathrm{~b}$ neurons were characterized at -60 $\mathrm{mV}$, where the burst-mediating T-type $\mathrm{Ca}^{2+}$ channels are most likely inactivated; Joksimovic et al., 2017); slicing angle and changes in intrinsic electrical properties when using a KMESO4-based intracellular solution as opposed to a KGLUC-based intracellular solution in our case (Kaczorowski et al., 2007).

Recent studies have extended the parameter space for splitting aPCx layer 2 neurons: long-range tracing studies identified layer-specific differences in axonal projection patterns (Chen et al., 2014; Diodato et al., 2016; Mazo et al., 2017). Different excitatory cell types have also been identified at the level of genetic markers. Genetic marker expression profiles of superficial neurons constituting layer 2a further support the "hard" location-based segregation principle applied here, as, based on published representative examples, they clearly seem to delineate a layer2a/layer2b border (Diodato et al., 2016; Choy et al., 2017; Bolding et al., 2019).

\section{Critical period imprinting and pruning of layer $\mathbf{2 b}$ neuron dendrites in the non-topographic aPCx}

The aPCx is a sensory brain region with a simple and evolutionary conserved structure that predates the development of the sensory neocortex (Bekkers and Suzuki, 2013). A central difference between sensory neocortex (auditory, visual, and somatosensory) and the aPCx is the spatial organization of afferent input. Sensory neocortex is a topographic circuit: nearby peripheral neurons share similar and predictable representations of sensory space and contact neighboring cortical neurons. These neighboring neurons have a high degree of local connectivity. In contrast, the PCx is a distributed, non-topographic circuit, where both the representation of sensory space and the recurrent connectivity are unpredictable and dispersed (Srinivasan and Stevens, 2018). This diffuse organization of the palaeocortical PCx is likely to reflect the primordial structure of cerebral cortices in reptiles before the evolution of isocortex in synapsids and later mammals (Fournier et al., 2015). These differences in the afferent input structure could affect dendritic growth patterns in palaeocortex and neocortex. In the following, we would therefore like to compare our data with published neocortical patterns of dendritic growth.

The determination of branch complexity during the first postnatal week corresponds to dendritic growth patterns identified in layer 2/3 and layer 5 pyramidal neurons in rodent neocortex (Petit et al., 1988; Maravall et al., 2004; Romand et al., 2011). The determination of branch complexity in the first postnatal week can therefore be considered a common design principle of neocortex and palaeocortex.

As in our palaeocortical dataset, length increase of neocortical dendrites was significant between the first and second postnatal week both in basal and apical dendrites. In the neocortex, there was no further increase in the period until p30 (Petit et al., 1988; Romand et al., 2011). The developmental trajectories of neocortex and palaeocortex diverge in developmental phase 3 during the critical period of sensory plasticity in the aPCx (between p12-p14 and $>$ p30): in the palaeocortical aPCx, we observed a reduction in total dendric length and in dendritic branch number in apical and basal dendrites between p12-p14 and p30. In neocortical layer 5 neurons, a decrease in branch number was observed earlier, before the onset of the critical period between p7 and p14 in apical but not in basal dendrites and was attributed to filopodial, not dendritic pruning (Romand et al., 2011).

We propose that an overabundance of dendrites at the beginning of the critical period in aPCx could be a consequence of the requirements of a non-topographic afferent input structure. Overabundance of distal apical layer 1a dendrites increases the combinatorial space for different glomerular input combinations. A surplus of basal dendrites results in more recurrent connections. We would have a larger probability of generating circuit motifs of recurrently connected neurons sharing similar sensory inputs. The enlarged combinatorial space maybe necessary in a distributed circuit like the PCx. In contrast, the topographic neocortex displays local clustering of similar inputs. This will result in a higher probability of the clustering of similar inputs on interconnected neurons. An effect of neocortical topographical organization may therefore be the effective wiring of significant features of sensory space. This would obviate the need for the metabolically expensive generation of superfluous dendritic branches during neocortical development. In the palaeocortical aPCx, the overabundance of dendrites combined with pruning could therefore enhance the initial combinatorial space and compensate for the lack of effective wiring by topography biases. Interestingly, the time window of pruning in layer $1 \mathrm{a}$ matches the critical period of NMDAR-dependent circuitspecific sensory synaptic plasticity in layer 1a (Franks Kevin and Isaacson, 2005; Poo and Isaacson, 2007). We therefore propose that in layer $2 \mathrm{~b}$ neurons, the critical period in the aPCx is accompanied by circuit-specific pruning 
and remodeling of the distal apical dendritic tree receiving sensory inputs in layer 1a. In the future, it will be interesting to test whether initial dendritic overabundance followed by pruning is a general feature of non-topographically ordered cortices.

\section{Possible contribution of supralinear dendritic integration to pruning}

In layer $2 b$ neurons, the enlargement of the olfactory coding space at the beginning of the critical period is followed by a reduction of dendritic branches. Here, we hypothesize that dendritic NMDAR-dependent supralinear stimulus-response behavior could serve as an underlying mechanism for this pruning process. Our rationale is that assuming constant synaptic density in layer $1 \mathrm{a}$, longer dendritic branches will result in a higher probability for regenerative NMDA-spikes evoked by clustered inputs. This is backed by a recent publication demonstrating that inputs clustered on the same layer 1 a branch in aPCx result in supralinear integration regardless of the distance between the inputs. In addition, large NMDAR-mediated $\mathrm{Ca}^{2+}$ signals occur during NMDA-spikes (Kumar et al., 2018). NMDAR-mediated $\mathrm{Ca}^{2+}$ signals are generally considered to promote dendritic growth during development (Konur and Ghosh, 2005). Our data therefore are compatible with an NMDA-spike-dependent selection and optimization process for apical dendritic layer $1 \mathrm{a}$ branches of layer $2 \mathrm{~b}$ neurons. NMDA-spikes occur with a higher probability on longer branches, the related $\mathrm{Ca}^{2+}$ rise would serve as a dendritotrophic signal. Such a mechanism could promote the observed survival and elongation of long branches at the expense of short branches. The result would be the structural self-amplification of dendritic NMDA-spikes by promoting branches with a higher probability for clustered inputs.

Although our $\mathrm{Ca}^{2+}$ imaging data in layer 1a strongly suggest local dendritic NMDA-spikes in layer 1a, our experiments do not exclude a significant contribution of recurrent inputs to the enlarged EPSP amplitudes we interpret as dendritic NMDA-spikes. With respect to pattern completion in the aPCx, such a mechanism is plausible. Supralinear stimulus-response behavior of layer 1a input evoked by coincident sensory and recurrent inputs recruited by our extracellular stimulation would also generate large local $\mathrm{Ca}^{2+}$ transients in the activated dendritic branches. This would both be the case when the inputs share the same (long) branch and project onto different branches. It is therefore also possible that supralinear stimulus-response behavior could be based on coincident activity between distal and proximal dendritic compartments. The supralinear integration of sensory and recurrent inputs could serve as a plasticity inducing coincidence detection mechanism. This mechanism would be comparable to the plateau potential evoked by coincident proximal and distal inputs in hippocampal CA1 pyramidal neurons (Takahashi and Magee, 2009). In the aPCx, such a mechanism would amplify synaptic connections between ensembles of recurrently connected neurons that share similar sensory inputs. Interestingly, we also observed an overabundance of the recurrently connected basal dendrites in layer $2 \mathrm{~b}$ neurons. These basal dendrites underwent pruning together with layer 1a dendrites. It is tempting to speculate that pruning skips apical and basal branches of interconnected layer $2 b$ neurons sharing similar sensory inputs. This could optimize the input-output function of dendritic branches coding for relevant features in interconnected neurons sharing sensory and recurrent inputs. Ideally, longitudinal in vivo studies of dendritic $\mathrm{Ca}^{2+}$ dynamics and the related dendritic growth during the critical period would be needed to further support this hypothesis.

In contrast, layer 2a neuron apical dendrites in layer 1a do neither display supralinear stimulus-response behavior of layer 1a inputs nor pruning. In addition, they have a larger dendritic tree in the sensory layer 1a. This fits well with the stronger incorporation into sensory circuits these neurons display (Suzuki and Bekkers, 2011; Wiegand et al., 2011) and may result in larger olfactory receptive fields that are developmentally hardwired.

In sum, we provide evidence for circuit-specific mechanisms of dendritic development in the aPCx. We demonstrated that different developmental trajectories of the dendritic tree in layer $2 \mathrm{a}$ and layer $2 \mathrm{~b}$ neurons relate to differences in circuit incorporation. Our data therefore support the concept that structural and functional differences between layer $2 a$ and layer $2 b$ neuron dendritic trees determine their distinct functions in the aPCx.

\section{References}

Bekkers JM, Suzuki N (2013) Neurons and circuits for odor processing in the piriform cortex. Trends Neurosci 36:429-438.

Bolding KA, Nagappan S, Han B, Wang F, Franks KM (2019) Pattern recovery by recurrent circuits in piriform cortex. bioRxiv 51:418458.

Chen CF, Zou DJ, Altomare CG, Xu L, Greer CA, Firestein SJ (2014) Nonsensory target-dependent organization of piriform cortex. Proc Natl Acad Sci USA 111:16931-16936.

Choy JMC, Suzuki N, Shima Y, Budisantoso T, Nelson SB, Bekkers JM (2017) Optogenetic mapping of intracortical circuits originating from semilunar cells in the piriform cortex. Cereb Cortex 27:589601.

Demir R, Haberly LB, Jackson MB (2001) Epileptiform discharges with in-vivo-like features in slices of rat piriform cortex with longitudinal association fibers. J Neurophysiol 86:2445-2460.

Diodato A, de Brimont MR, Yim YS, Derian N, Perrin S, Pouch J, Klatzmann D, Garel S, Choi GB, Fleischmann A (2016) Molecular signatures of neural connectivity in the olfactory cortex. Nat Commun 7:12238.

Feng L, Zhao T, Kim J (2015) Neutube 1.0: a new design for efficient neuron reconstruction software based on the swc format. eNeuro 2:ENEURO.0049-14.2014.

Fournier J, Müller CM, Laurent G (2015) Looking for the roots of cortical sensory computation in three-layered cortices. Curr Opin Neurobiol 31:119-126.

Franks Kevin M, Isaacson JS (2005) Synapse-specific downregulation of NMDA receptors by early experience: a critical period for plasticity of sensory input to olfactory cortex. Neuron 47:101-114.

Goebbels S, Bormuth I, Bode U, Hermanson O, Schwab MH, Nave K-A (2006) Genetic targeting of principal neurons in neocortex and hippocampus of NEX-Cre mice. genesis 44:611-621.

Haberly LB (1985) Neuronal circuitry in olfactory cortex: anatomy and functional implications. Chem Senses 10:219-238.

Hagiwara A, Pal SK, Sato TF, Wienisch M, Murthy VN (2012) Optophysiological analysis of associational circuits in the olfactory cortex. Front Neural Circuits 6:18. 
Ho J, Tumkaya T, Aryal S, Choi H, Claridge-chang A (2019) Moving beyond $P$ values: data analysis with estimation graphics. Nat Methods 16:565-566.

Hoffpauir BK, Marrs GS, Mathers PH, Spirou GA (2009) Does the brain connect before the periphery can direct?: a comparison of three sensory systems in mice. Brain Res 1277:115-129.

Johenning FW, Beed PS, Trimbuch T, Bendels MHK, Winterer J, Schmitz D (2009) Dendritic compartment and neuronal output mode determine pathway-specific long-term potentiation in the piriform cortex. J Neurosci 29:13649-13661.

Joksimovic SM, Eggan P, Izumi Y, Joksimovic SL, Tesic V, Dietz RM, Orfila JE, DiGruccio MR, Herson PS, Jevtovic-Todorovic V, Zorumski CF, Todorovic SM (2017) The role of T-type calcium channels in the subiculum: to burst or not to burst? J Physiol 595:6327-6348.

Kaczorowski CC, Disterhoft J, Spruston N (2007) Stability and plasticity of intrinsic membrane properties in hippocampal CA1 pyramidal neurons: effects of internal anions. J Physiol 578:799-818.

Kanari L, Ramaswamy S, Shi Y, Morand S, Meystre J, Perin R, Abdellah M, Wang Y, Hess K, Markram H (2019) Objective morphological classification of neocortical pyramidal cells. Cereb Cortex 29:1719-1735.

Konur S, Ghosh A (2005) Calcium signaling and the control of dendritic development. Neuron 46:401-405.

Kumar A, Schiff O, Barkai E, Mel BW, Poleg-Polsky A, Schiller J (2018) NMDA spikes mediate amplification of inputs in the rat piriform cortex. Elife 7:e38446.

Lanoue V, Cooper HM (2018) Branching mechanisms shaping dendrite architecture. Dev Biol 451:1-9.

Leighton AH, Lohmann C (2016) The wiring of developing sensory circuits-from patterned spontaneous activity to synaptic plasticity mechanisms. Front Neural Circuits 10:219.

Luskin MB, Price $\mathrm{J}$ (1983) The laminar distribution of intracortical fibers originating in the olfactory cortex of the rat. $\mathrm{J}$ Comp Neurol 216:292-302.

Madisen L, Garner AR, Shimaoka D, Chuong AS, Klapoetke NC, Li L, van der Bourg A, Niino Y, Egolf L, Monetti C, Gu H, Mills M, Cheng A, Tasic B, Nguyen TN, Sunkin SM, Benucci A, Nagy A, Miyawaki A, Helmchen F, et al. (2015) Transgenic mice for intersectional targeting of neural sensors and effectors with high specificity and performance. Neuron 85:942-958.

Maravall M, Koh IYY, Lindquist WB, Svoboda K (2004) Experiencedependent changes in basal dendritic branching of layer $2 / 3$ pyramidal neurons during a critical period for developmental plasticity in rat barrel cortex. Cereb Cortex 14:655-664.

Martin-Lopez E, Ishiguro K, Greer CA (2019) The laminar organization of piriform cortex follows a selective developmental and migratory program established by cell lineage. Cereb Cortex 29:1-16.

Mazo C, Grimaud J, Shima Y, Murthy VN, Geoffrey Lau C (2017) Distinct projection patterns of different classes of layer 2 principal neurons in the olfactory cortex. Sci Rep 7:8282.

Pachitariu M, Stringer C, Schröder S, Dipoppa M, Rossi LF, Carandini M, Harris KD (2017) Suite2p: beyond 10,000 neurons with standard two-photon microscopy. bioRxiv. doi:https://doi. org/10.1101/061507.

Petit TL, LeBoutillier JC, Gregorio A, Libstug H (1988) The pattern of dendritic development in the cerebral cortex of the rat. Brain Res 469:209-219.

Poirazi P, Brannon T, Mel BW (2003) Pyramidal neuron as two-layer neural network. Neuron 37:989-999.

Poo C, Isaacson JS (2007) An early critical period for long-term plasticity and structural modification of sensory synapses in olfactory cortex. J Neurosci 27:7553-7558.

Rigas P, Adamos DA, Sigalas C, Tsakanikas P, Laskaris NA, Skaliora I (2015) Spontaneous Up states in vitro: a single-metric index of the functional maturation and regional differentiation of the cerebral cortex. Front Neural Circuits 9:219.

Romand S, Wang Y, Toledo-Rodriguez M, Markram H (2011) Morphological development of thick-tufted layer $\mathrm{V}$ pyramidal cells in the rat somatosensory cortex. Front Neuroanat 5:1-27.

Rueckl M, Lenzi SC, Moreno-Velasquez L, Parthier D, Schmitz D, Ruediger S, Johenning FW (2017) SamuROI, a Python-based software tool for visualization and analysis of dynamic time series imaging at multiple spatial scales. Front Neuroinform 11:10141059.

Sarma AA, Richard MB, Greer CA (2011) Developmental dynamics of piriform cortex. Cereb Cortex 21:1231-1245.

Scorcioni R, Polavaram S, Ascoli GA (2008) L-Measure: a web-accessible tool for the analysis, comparison and search of digital reconstructions of neuronal morphologies. Nat Protoc 3:866-876.

Sommer C, Straehle C, Kothe U, Hamprecht FA (2011) llastik: interactive learning and segmentation toolkit. Eighth IEEE International Symposium on Biomedical Imaging (ISBI 2011) Proceedings, pp 230-233. Chicago, IL: IEEE.

Srinivasan S, Stevens CF (2018) The distributed circuit within the piriform cortex makes odor discrimination robust. J Comp Neurol 526:2725-2743.

Suzuki N, Bekkers JM (2006) Neural coding by two classes of principal cells in the mouse piriform cortex. J Neurosci 26:1193811947.

Suzuki N, Bekkers JM (2010) Inhibitory neurons in the anterior piriform cortex of the mouse: classification using molecular markers. J Comp Neurol 518:1670-1687.

Suzuki N, Bekkers JM (2011) Two layers of synaptic processing by principal neurons in piriform cortex. J Neurosci 31:2156-2166.

Takahashi H, Magee JC (2009) Pathway interactions and synaptic plasticity in the dendritic tuft regions of CA1 pyramidal neurons. Neuron 62:102-111.

Torben-Nielsen B (2014) An efficient and extendable Python library to analyze neuronal morphologies. Neuroinformatics 12:619-622.

Wiegand HF, Beed P, Bendels MHK, Leibold C, Schmitz D, Johenning FW (2011) Complementary sensory and associative microcircuitry in primary olfactory cortex. J Neurosci 31:1214912158.

Wilson DA, Sullivan RM (2011) Cortical processing of odor objects. Neuron 72:506-519. 